\title{
Confusion in differential piston measurement with the pyramid wavefront sensor
}

\author{
A. Bertrou-Cantou ${ }^{1}$, E. Gendron ${ }^{1}$, G. Rousset ${ }^{1}$, V. Deo ${ }^{2}$, F. Ferreira $^{1}$, A. Sevin ${ }^{1}$, and F. Vidal ${ }^{1}$ \\ ${ }^{1}$ LESIA, Observatoire de Paris, Univ. PSL, CNRS, Sorbonne Univ., Université de Paris, 5 place Jules Janssen, 92195 Meudon, \\ France \\ e-mail: arielle. bertrou@obspm. fr \\ 2 National Astronomical Observatory of Japan, Subaru Telescope, 650 North A'ohoku Place, Hilo, HI 96720, USA
}

Received 25 June 2021 / Accepted 4 October 2021

\begin{abstract}
Context. The pyramid was proven to be a highly sensitive and versatile wave-front sensor (WFS) and has been selected to be installed on the single conjugate adaptive optics (AO) systems of the extremely large telescope (ELT). The pupil of the ELT is fragmented by the secondary support spider arms, which are larger than the spatial coherence length of the atmospheric turbulence. This causes a rupture of the incoming wavefront continuity, which means that we need to be able to measure the differential pistons across the spider arms to achieve full wavefront reconstruction.

Aims. We investigate the reaction of the modulated pyramid WFS to discontinuous aberrations in presence of phase residuals after AO compensation for a range of expected observing conditions at the location of the ELT. We then explore some parameters of the sensor in order to improve its sensitivity to the wavefront discontinuities, including optical gain compensation, specific sensor modulation paths, and the number of faces of the pyramidal prism.

Methods. We derived sensitivity loss and modal cross-talk strength coefficients around static post-AO residual phases using the COMPASS end-to-end AO simulation software. Moreover, extensive closed-loop AO simulations let us derive two wavefront error criteria that are appropriate for a fragmented pupil geometry. We used these to assess the wavefront reconstruction performance.

Results. We show that on the ELT, the pyramid experiences a drastic loss in sensitivity and also non-linear modal cross-talks. Added to the limited capture range, this makes it poorly suited for phase discontinuity measurements at visible wavelengths. The strategies we studied to increase the sensitivity and reduce the modal cross-talk provide an improvement of the reconstruction for low $D / r_{0}\left(\lambda_{\mathrm{WFS}}\right)$ values, that is, for a $K$-band pyramid. In presence of a large residual wavefront variance, however, a similar sensor in visible light fails to provide the necessary trade-off to measure both the continuous modes and the wavefront discontinuities.

Conclusions. The ELT instruments, designed with only visible-band pyramid WFSs, will not be able to perform a direct measurement of the wavefront discontinuities. They will have to rely on Kolmogorov statistics to restore the continuity of the atmospheric corrugated wavefront. If any other source of discontinuities arises on the ELT, instruments will need an additional, dedicated WFS.
\end{abstract}

Key words. instrumentation: adaptive optics - telescopes

\section{Introduction}

The ability of adaptive optics (AO) to compensate for atmospheric distorted wavefronts and to enable a diffraction-limited beam (or close to it) is now demonstrated on every large groundbased optical telescope. This technology has matured rapidly over the past three decades, and the AO community is currently paving the way for the class of extremely large telescopes, ranging from $20 \mathrm{~m}$ diameter and larger. Their preparation goes hand in hand with new challenges that must be addressed to ensure the effective operation of AO on sky. In the context of the Extremely Large Telescope (ELT, Tamai et al. 2016) of the European Southern Observatory (ESO), the fragmentation of the telescope pupil by the spider arms was identified as a major concern, with critical impacts on the overall AO performance. An adequate compensation of the wavefront will only be achieved depending on the capability of a given $\mathrm{AO}$ design to provide a cophased wavefront over the pupil fragments.

The ELT is equipped with a six-armed $51 \mathrm{~cm}$ wide spider to support its secondary mirror. While the arms provide the necessary structural stiffness, their width is larger than the spatial coherence length $r_{0}$ of the perturbed wavefront, which is typi- cally $10-15 \mathrm{~cm}$ at $500 \mathrm{~nm}$ at the ELT site of Cerro Armazones. It results in a significant fragmentation of the optical pupil and in a break of the spatial continuity of the incoming wavefront phase.

Therefore the ELT consists of six fragments of equal areas delimited by the spider arms. We call the average phase value measured over a fragment a petal. The petal modes are to each piece of the pupil what the piston mode is to the full pupil. We call the petal difference between a pupil fragment a reference fragment the differential piston $(\Delta P)$. These degrees of freedom should be considered as modes (or linear combination of modes) to be sensed and compensated for as well as any other continuous mode. They will be part of the control space of the deformable mirror (DM) and should not be filtered out to reconstruct the full wavefront: simply think of astigmatism, a continuous mode that comes with built-in petals, that is, has a non-zero average value on given fragments of the pupil.

On the other hand, the deformable mirror installed in the telescope unit is composed of six independent adaptive shells that follow the fragmented pupil geometry. The wavefront shape arising from the action of a single actuator, that is, the influence function (IF), is defined within each sub-DM shell only and is cropped at the spider level: an actuator at an edge of a spider 
does not affect the wavefront at the other edge, meaning that each sub-DM can produce a pure petal mode.

The ELT can be seen as an array of six sub-telescopes that need to be co-phased at the AO loop frequency with a suitable wavefront sensor (WFS) in order to compensate for the atmospheric $\Delta P$ and to work as a single telescope with a diameter of $38.5 \mathrm{~m}$. Then, a full reconstruction of the wavefront including the phase steps across these spider gaps is an absolute prerequisite to ensure that the telescope can deliver its full scientific benefits.

The pyramid wavefront sensor (P-WFS) is currently turning into the preferred design for post-2020 AO systems. It was introduced by Ragazzoni (1996), and the AO community became interested in it for its high sensitivity and versatility compared to Shack-Hartmanns and pushed it forward, making it a firstchoice candidate in the context of extremely large telescopes despite its non-linear properties (Vérinaud 2004; Burvall et al. 2006; Esposito et al. 2000b).

The P-WFS was studied and considered as a phasing sensor in the laboratory (Esposito et al. 2000a) and on-sky for the ELT segment co-phasing (Pinna et al. 2008) as well as for differential piston measurement in stellar interferometry at the Large Binocular Telescope (Vérinaud \& Esposito 2002). However, the ability to sense the phase steps across the spider arms at AO loop frequency and under the closed-loop AO residuals encountered at ELT is now being severely questioned. The two first-light instrument HARMONI (Thatte et al. 2021) and MICADO (Davies et al. 2018) single-conjugate AO (SCAO) modes (Sauvage et al. 2018; Clénet et al. 2018) highlighted miscorrections of the petal modes when a P-WFS was used at visible wavelengths, causing the so-called island effect, which is an amplification of the petal mode error and strongly affects the imaging quality of the scientific path (Schwartz et al. 2018; Bertrou-Cantou et al. 2019). For instance, the expected Strehl ratio (SR) for a continuous pupil and a Fried parameter of $r_{0}(500 \mathrm{~nm})=12.8 \mathrm{~cm}$ is $82 \%$ in $K$ band on the ELT, but it drops down to about $20 \%$ in presence of the island effect.

To cope with this effect, suggested solutions rely on the continuity of turbulence-induced wavefronts. We can cite the pairing method, which consists of coupling the actuators facing each other at either edge of the spiders and enforce them to take the same position (Schwartz et al. 2018). Along the same line of preventing discontinuities at the spider level, a modal basis was introduced in Bertrou-Cantou et al. (2020), enabling us to reach $78 \%$ of $K$-band SR in the same previous conditions. We can also mention the minimum mean-squares error (MMSE) reconstructor proposed in Hutterer et al. (2018) and in Bertrou-Cantou et al. (2019), which uses the assumption of Kolmogorov laws of turbulence as a prior to infer the best estimated petals to compensate for the DM.

Nevertheless, these algorithmic solutions have several drawbacks. They are only valid under the assumptions of atmospherically induced wavefronts and no longer hold for other sources of errors, such as the low-wind effect (LWE, Milli et al. 2018), thermo-mechanical drifts of the DM shells, and primarymirror segment misphasing. Moreover, the continuity hypothesis encounters limitations in poor atmospheric conditions (typically above 1 arcsec seeing) because the phase amplitudes across the spider gaps increase with the seeing. Some authors therefore deem a physical optical measurement of petals to be more reliable: focal-plane wavefront-sensing techniques are currently studied for 8-m class telescopes to deal with LWE (N'Diaye et al. 2018; Vievard et al. 2019). Moreover, unlike the results of MICADO and HARMONI, Hippler et al. (2019) reported a perfect correction of petal modes using a pyramid sensor operated in $K$ band in the frame of the study of the METIS instrument. METIS was the first-light mid- to thermal infrared spectro-imager for the ELT (Brandl et al. 2018). This result gives some hope for the sensing capabilities of differential pistons by a pyramid sensor.

The aim of this paper is twofold. First, we investigate under which conditions the P-WFS still has the ability to sense the petal modes in addition to the residual phase distortions. Second, we aim to understand the process of petal divergence in the wavefront reconstruction that causes the island effect and to identify strategies and observation regimes that minimise this effect.

These two aspects are studied at the ELT scale using the endto-end simulation software COMPASS (Ferreira et al. 2018), which we detail in Sect. 2. We study in Sect. 3 the response of the P-WFS to petal modes in a diffraction-limited regime and the variation of this response with closed-loop AO residuals. In Sect. 4 we further study the pyramid non-linearity and instabilities in the particular case of fragmented pupils, and we highlight limitations that go beyond the classical framework of pyramid non-linearity compensation. Section 5 investigates possible strategies to enhance the pyramid sensitivity to petals: we explore non-circular modulation patterns and perform simulations for an extensive range of $D / r_{0}\left(\lambda_{\mathrm{WFS}}\right)$ ratios covering visible and infrared wavelengths for wavefront sensing at ELT. Finally, we discuss limitations and perspectives of our simulation results in Sect. 6.

\section{AO simulation environment}

\subsection{Simulation conditions}

Running simulations in ELT-like conditions requires a powerful and efficient AO simulation platform, for which COMPASS has been developed (Ferreira et al. 2018). This end-to-end simulation tool allows us to perform numerical simulations with the set-up and conditions summarised in Table 1.

The telescope pupil is a dodecagonal mirror with a diameter of $38.5 \mathrm{~m}$ made of 798 hexagonal joined segments, with a secondary mirror obstruction with a diameter of $11.1 \mathrm{~m}$. These diameter definitions stand for an unvignetted beam with a $10^{\prime}$ field of view (Dierickx \& McPherson 2012). We assume that the segments are perfectly co-phased and contiguous so that their assembly acts as a monolithic primary mirror. The spider supporting the secondary mirror has six arms of $51 \mathrm{~cm}$ thickness. In this paper, the results are obtained considering the primary mirror as defined by ESO and the spider as the pupil.

The total number of actuators of the DM is 5352, which yields 892 actuators in each shell. The projected DM size onto the pupil plane is larger than the pupil: four rows of actuators can extend the wavefront correction beyond the clear on-axis aperture. For this reason, we introduce the notion of active actuators in Appendix A. They are the DM degrees of freedom.

The DM actuators follow a triangular pattern with $54 \mathrm{~cm}$ pitch in the primary pupil plane (Jochum et al. 2008). We configured COMPASS to use a custom-defined DM IF, provided to us by ESO. These were obtained from a mechanical finiteelement analysis of the DM shell and were re-sampled for the purpose of AO simulations. IFs are designed with null coupling: while the extent of the IF is larger than one DM pitch, its value at the location of any other actuator is zero. Moreover, their combination allows a perfectly flat, pure petal mode to be generated because they originate from the deformation of a flat, free-floating plate. This important property would not be 
Table 1. Configuration used for the COMPASS AO simulations.

\begin{tabular}{|c|c|}
\hline \multicolumn{2}{|r|}{ Simulation configuration } \\
\hline Pupil & $\begin{array}{c}\text { ELT pupil }{ }^{(a)} \\
\text { Monolithic primary mirror } \\
\text { six-armed } 51 \mathrm{~cm} \text {-thick spider } \\
\text { Central obstruction } 11.1 \mathrm{~m}\end{array}$ \\
\hline Turbulence & $\begin{array}{c}\text { Single ground layer } \\
r_{0}=\{7.6-76.0\} \mathrm{cm} \text { at } 500 \mathrm{~nm} \\
\text { Outer scale } L_{0}=25 \mathrm{~m} \\
\text { Wind speed }|\boldsymbol{v}|=10 \mathrm{~m} . \mathrm{s}^{-1}\end{array}$ \\
\hline DM & $\begin{array}{l}5352 \text { actuators for the } 6 \text { shells }{ }^{(b)} \\
\text { Triangular pattern per shell } \\
54 \mathrm{~cm} \text { pitch } \\
\text { ESO provided influence functions }{ }^{(c)} \\
\text { Null coupling between actuators }\end{array}$ \\
\hline P-WFS & $\begin{array}{c}r_{\text {mod }}=3 \frac{\lambda}{D} \\
\lambda_{\mathrm{WFS}}=700 \mathrm{~nm} \\
95 \times 95 \text { sub-apertures } \\
\text { No noise (photon and read-out) }\end{array}$ \\
\hline Target & $\begin{array}{c}\lambda_{\mathrm{tar}}=1100 \mathrm{~nm} \\
\text { On-axis star }\end{array}$ \\
\hline Controller & $\begin{array}{c}\text { Loop rate } 500 \mathrm{~Hz} \\
\text { No latency (except integration and hold) } \\
\text { Linear modal integrator }\end{array}$ \\
\hline
\end{tabular}

Notes. ${ }^{(a)}$ Dierickx \& McPherson (2012), ${ }^{(b)}$ Jochum et al. (2008), ${ }^{(c)}$ Bonnet (priv. comm.).

met using a linear combination of identical theoretical Gaussian IFs, which is a classical approximation that is often proposed in AO simulation software packages. In this case, a least-squares approximation of petal modes would indeed generate some significant ringing near the edges. This ringing is measured by the P-WFS as the signature of the petal mode, which in turn completely biases the study. It is therefore important to feature those very specific DM influence functions. The shape of one of the six ELT pupil fragments and the DM actuators layout is presented in Fig. A.1

The WFS is a four-sided pyramid that operates in the visible around $700 \mathrm{~nm}$ and samples the pupil with $95 \times 95$ sub-apertures (detector pixels), that is, a $40 \mathrm{~cm}$ pitch projected in the primary mirror plane. It operates with a typical modulation of $3 \frac{\lambda}{D}$ in the range identified to provide a reasonable performance trade-off at all magnitudes for the MICADO SCAO design (Vidal et al. 2017).

P-WFS output measurements are defined in the "full pixel" approach: normalised pixel values that lie within the four pupil images. A reference value is subtracted to obtain a null signal for a null incoming phase. This global measurement approach is inherited from Deo et al. (2018) and proved to provide identical or better end-to-end performance than the original P-WFS "slopes map" control. Pixels that lie underneath the spiders' shadow have been kept in the measurement computation as they bear specific importance in the sensing of differential pistons (Vérinaud \& Esposito 2002). We use the term "slopes" to refer to the pixel measurements for the sake of $\mathrm{AO}$ naming conventions.

A single-layer turbulence is generated from von Kármán statistics, with $r_{0}$ values ranging from $7.6 \mathrm{~cm}$ to $76 \mathrm{~cm}$, expressed at $500 \mathrm{~nm}$, and the outer scale is $L_{0}=25 \mathrm{~m}$. The pupil-plane sampling of the simulation is taken as the smallest power of 2, yielding at least 3 pixels per $r_{0}$.

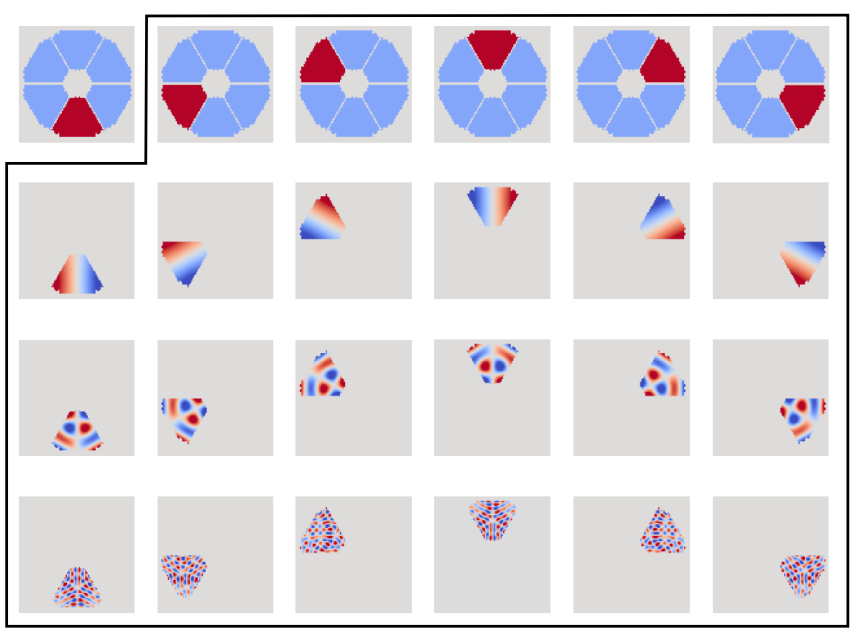

Fig. 1. Modes of the basis $\boldsymbol{B}_{\text {petal }}$ used in Sect. 4: Six petal modes, and a few intra-petal modes. The modes are displayed over the primary mirror pupil. The modes enclosed in black are used to characterise the sensing properties of the P-WFS.

The AO loop runs without latency, meaning that the correction is applied on the DM during the frame immediately following the measurement. Data transfer, computation, and DM rise time are not considered.

\subsection{Modal basis compatible with the study of pupil fragmentation}

To investigate the properties of the P-WFS response to petal modes, we first need to introduce an appropriate modal basis $\boldsymbol{B}_{\text {petal }}$ that explicitly expresses petal modes to specifically address and characterise their properties. This basis is orthogonal in the DM command space to ensure that the confusion between the modes we study reflects a confusion in the P-WFS measurements and not from the command space. Plus, it is orthogonal to pure global piston, a mode which does not add phase information and to which the P-WFS is blind.

To do so, we computed six modal basis defined over each shell of the DM, following the process of modal basis computation over the active actuators and then extending the modes to the passive ones, as described in the Appendices A-C. The concatenation of the six bases defined per shell provided a single basis (see Fig. 1) expressing the six petal modes $\phi_{i<6}$ and modes orthogonal to them, referred to as intra-petal modes and noted $\phi_{i \geq 6}$. The petal modes are defined as

$\phi_{i}(x, y)= \begin{cases}1 \mathrm{rad} & \text { if }(x, y) \in i \text { th fragment } \\ 0 & \text { elsewhere }\end{cases}$

with $(x, y)$ being the pupil coordinates and $i$ the index of the six DM shells. The intra-petal modes are normalised in terms of phase and ordered by increasing spatial frequency. This gives a basis that is not orthogonal to pure global piston, but of which petal modes are explicit orthogonal members. We can filter the first mode $\phi_{0}$ from the basis because it can be expressed as a linear combination of the five others $\phi_{1-5}$ and the global piston mode. However, we kept track of this mode in the simulations because it is further used as reference in Eq. (11) and is necessary to infer the petal error in Eq. (17). This modal basis is used in Sect. 4 for the sole purpose of investigating the pyramid response to $\Delta P$. Unless otherwise stated, the expressions using the $\phi_{i}$ and their resultants stand for the petal basis $\boldsymbol{B}_{\text {petal }}$. 

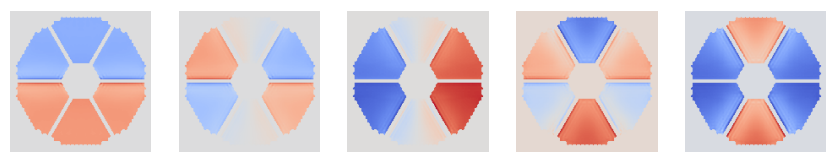

Fig. 2. Last eigenvectors of the interaction matrix: they are dominated by petal modes.

In Sect. 5 we report the results we obtained with closed AO loops. For convenience, we make use of a second modal basis that was directly computed over the active actuators of the overall DM (Appendix B). Once again, we follow the process detailed in Appendix C. The modes defined over the full DM (not the DM shells) are shown in Fig. C.1.

\section{Differential piston measurement with the P-WFS}

In this section, we investigate the P-WFS response to differential pistons during calibration and during operation. Calibration refers to a measurement done at the diffraction limit while the measurement in operation is done around phase residuals left by the AO system and specific to the seeing conditions.

\subsection{Calibration}

\subsubsection{Interaction matrix}

The calibration of the interaction matrix $\boldsymbol{D}$ aims to characterise the linear part of the WFS response to each modal degree of freedom of the DM, usually for small variations around a flat wavefront at diffraction $\phi_{\mathrm{cal}}$. It characterises the linear relation between each mode of the DM $\phi_{i}$ and the WFS response, that is, the measurement vector $s$. To stay within the linear range of the pyramid, we applied small positive and negative pokes of amplitudes $\epsilon$ for each mode of the basis. The calibrated interaction matrix is thus defined using

$\boldsymbol{D}_{\mathrm{cal}}=\left\{\frac{\boldsymbol{s}\left(\epsilon \phi_{i}+\phi_{\mathrm{cal}}\right)-\boldsymbol{s}\left(-\epsilon \phi_{i}+\phi_{\mathrm{cal}}\right)}{2 \epsilon}\right\}_{0 \leq i \leq N}$.

From a singular-value decomposition, it stands out that the five weakest eigenvectors of $\boldsymbol{D}_{\text {cal }}$ form a subspace mostly made of a combination of petal modes (see Fig. 2). This means that petal modes are poorly sensed and that the P-WFS measurements bear little petal-sensing information.

The modal reconstructor $\boldsymbol{D}_{\mathrm{cal}}^{\dagger}$ is obtained using the pseudoinverse of the matrix $\boldsymbol{D}_{\text {cal }}$,

$\boldsymbol{D}_{\text {cal }}^{\dagger}=\left(\boldsymbol{D}_{\text {cal }}^{T} \boldsymbol{D}_{\text {cal }}\right)^{-1} \boldsymbol{D}_{\text {cal }}^{T}$.

The inversion is conditioned to preserve all degrees of freedom, including the five weak modes presented in Fig. 2, and such that the product of the modal reconstructor and the interaction matrix gives an identity matrix,

$\boldsymbol{D}_{\text {cal }}^{\dagger} \boldsymbol{D}_{\text {cal }}=$ Id.

The modal decomposition of WFS measurements for an incoming phase in operation $\phi_{\mathrm{op}}$ is contained in the expansion coefficients of the vector $\boldsymbol{a}$. It is derived from the pyramid measurement vector $s$ using the equation

$\boldsymbol{a}\left(\phi_{\mathrm{op}}\right)=\boldsymbol{D}_{\mathrm{cal}}^{\dagger} \boldsymbol{s}\left(\phi_{\mathrm{op}}\right)$.

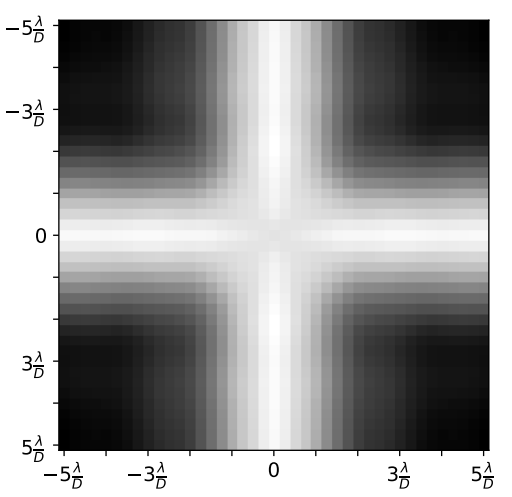

Fig. 3. Sum of the P-WFS sensitivity maps to each petal mode with respect to the position of the incoming beam. The highest-sensitivity light areas are found at the vertex and edges of the pyramid.

Finally, the estimated phase applied on the DM is the sum of all the basis modes $\phi_{i}$,

$\phi_{\mathrm{est}}(x, y)=\sum a_{i} \phi_{i}(x, y)$

\subsubsection{Sensitivity}

The modal sensitivity of the P-WFS to a mode $\phi_{i}$ is the Euclidean norm of the pyramid response $s$ to this mode,

$\operatorname{sensitivity}\left(\phi_{i}\right)=\left\|s\left(\phi_{i}\right)\right\|$.

In Fig. 3 we show a detailed map in which each pixels shows the sensitivity of the pyramid to petal modes for a nonmodulated diffraction-limited beam centred at that pixel location in the pyramid focal plane. We summed the Euclidean norm of the P-WFS response of the six modes for any pixel, that is, any beam position covering $-5 \frac{\lambda}{D}$ to $5 \frac{\lambda}{D}$ of the pyramid mask in the $x$-axis and $y$-axis directions. The white cross is aligned with the pyramid edges: a good sensitivity to the petals is found in the diffractive region of the pyramid, within up to $1 \frac{\lambda}{D}$ from the pyramid vertex and edges. The aberrated point spread function in this region is split and creates an interference signal below the spiders in the four pupils in the sensor plane. A small modulation radius (or no modulation) or a phase mask with a high number of diffractive elements (edges) will then be beneficial to sense these modes. These parameters are explored in Sect. 5.

\subsubsection{Dynamic range}

The algebra behind the computation of the modal reconstruction matrix $\boldsymbol{D}_{\text {cal }}^{\dagger}$ assumes the linearity of the WFS. However, the P-WFS exhibits a linear behaviour only around low phase values, and it is known to enter a saturation regime for higher values. This saturation starts to show up in orange on Fig. 4 for the mode $\phi_{100}$, orthogonal to petals, where the solid line depicts the reconstruction around the calibration regime $\phi_{\text {cal }}$. The extent of the dynamic range in which the linearity assumption is met certainly depends on the modulation value and on the spatial frequency of the mode. This type of behaviour applies to all modes except petal modes, which are exempt from the rule.

In contrast, the pyramid response for the petal mode $\phi_{1}$, plotted as the solid blue line, differs from the general case: It is sinusoidal with a period of $\lambda$. The reconstruction is then limited by the sensing wavelength and only provides meaningful results in the range of $]-\frac{\lambda}{2}, \frac{\lambda}{2}\left[\right.$. The behaviour in the range $\left[\frac{\lambda}{2}, \lambda[\right.$ will favor 


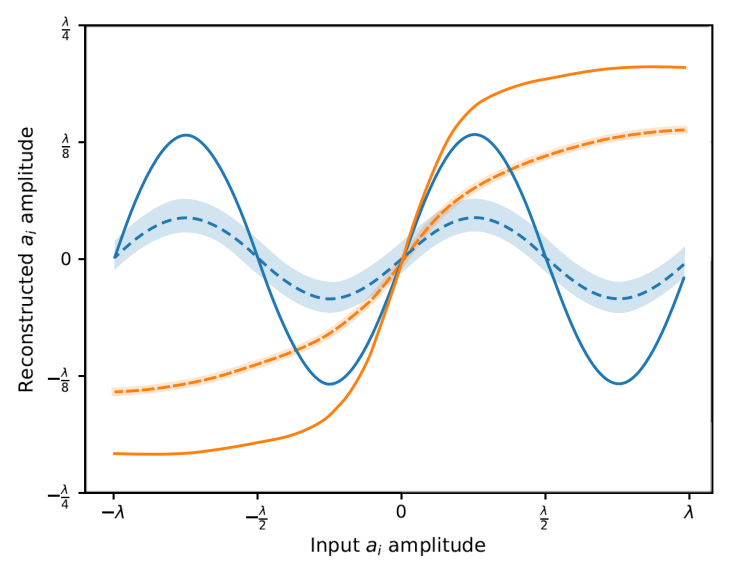

Fig. 4. Pyramid response to a petal mode $\phi_{1}$ (blue) and to a mode orthogonal to petals $\phi_{100}$ (orange) around calibration (plain line) and during operation (dashed line), i.e. around a residual wavefront $\phi_{\text {intra }}$ free of $\Delta P$ and obtained with a closed-loop system with $r_{0}=12.8 \mathrm{~cm}$ at $500 \mathrm{~nm}$. The response in operation is an average over ten independent residual wavefronts with a shaded $\pm 1 \sigma$ error bar.

the divergence of the servo loop. Hence, the P-WFS is inherently blind to the divergence of petal modes, as it reduces to an interferometric sensor subject to phase wrapping in that case (Esposito et al. 2000a). This interferometric interpretation is also expected to provide meaningful insights for broadband sensors beyond what we simulated for this work.

\subsection{Impact of $A O$ residuals}

\subsubsection{Residual phase description}

The main characteristics of the AO system are its loop frequency and the number of actuators, which define its spatial and temporal compensation bandwidths, respectively. Phase perturbations evolving beyond these limits are left uncompensated for by the system. Additional errors add upon these, such as noise, wavefront sensing errors and reconstruction errors, and petal divergence. This results in an imperfect correction of the corrugated phase, leaving AO loop residuals in the WFS and scientific paths. COMPASS simulations allow us to compute these residual wavefronts.

A typical example of such a residual phase $\phi_{\text {op }}$ found in operation is shown in Fig. 5, with the associated point spread function (PSF) computed at $1100 \mathrm{~nm}$. Each AO error term present in $\phi_{\mathrm{op}}$ is visible there: the smallest-scale structures are due to the $\mathrm{AO}$ fitting error term $\phi_{\text {fitting }}$, which is orthogonal to the DM space. Lower-order patterns that can be distinguished inside each pupil fragment are modal residuals $\phi_{i \geq 6}$ lying within the DM space (most likely temporal errors). We combine the fitting and modal residual errors under the same term intra-petal residuals, which we note

$\phi_{\text {intra }}=\phi_{\text {fitting }}+\sum_{i \geq 6} a_{i} \phi_{i}$.

Finally, the most visually striking effect in Fig. 5 is the petal mode residuals $\phi_{i<6}$. The residual wavefront is characterised by phase steps delimited by the spider arms. These differential pistons lead to the secondary PSF protuberances at $\sim 2 \lambda / \mathrm{D}$ of the central core of the observed PSF. Finally, we write the residual phase in operation as the sum of these two contributors,

$\phi_{\text {op }}=\phi_{\text {intra }}+\sum_{i<6} a_{i} \phi_{i}$ (a)

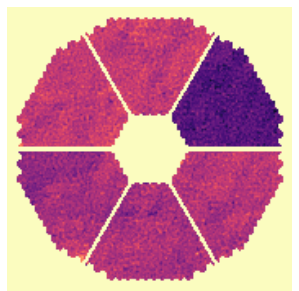

(b)

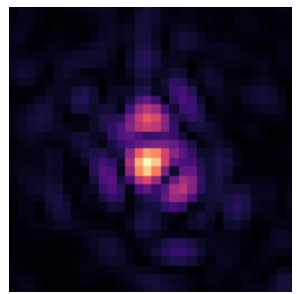

Fig. 5. Left: simulated residual wavefront error over the primary mirror. Right: PSF in presence of the island effect, obtained with a Fried parameter of $r_{0}(500 \mathrm{~nm})=12.8 \mathrm{~cm}$ and a pyramid in the visible wavelength. The island effect clearly appears and the SR is $28 \%$ at $1100 \mathrm{~nm}$, compared with $50 \%$ without the island effect.

\subsubsection{Pyramid response in operation}

We continue the analysis started in Sect. 3.1.3 of the pyramid response to an increasing petal mode amplitude in a diffractionlimited regime, and we extend it in this section to the regime with intra-petal residuals $\phi_{\text {intra }}$. The reconstruction of the petal mode $\phi_{1}$ in this operating regime is shown by the dashed blue line in Fig. 4. The residual phase $\phi_{\text {intra }}$ used to compute Fig. 4 was obtained in closed loop with the P-WFS and a seeing of 0.8 arcsec. The mean value and $1 \sigma$ error bar were obtained using ten independent draws of the residual phases. The P-WFS response variation between the diffraction limit and 0.8 arcsec data is characterised by two major effects. First, there is a significant loss in sensitivity compared to the response around a null phase $\phi_{\text {cal }}$, which is the expected P-WFS optical gain effect (e.g., Deo et al. 2019): The response is scaled with a coefficient $\alpha$. This sensitivity loss is also observed to a lesser extent for mode $\phi_{100}$ (dashed orange line).

The second response variation is more surprising: An additive offset $\beta$ appears over the reconstructed petal mode amplitude. This bias is independent of the amplitude of the input petal mode, it is constant for a given draw of $\phi_{\text {intra }}$, and specific to it. The effect of $\beta$ is visible in Fig. 4 through the $\pm 1 \sigma$ error bars (shaded area) that reflect its variations over the $10 \phi_{\text {intra }}$ draws. This observation is also valid for other intra-petal modes, but the variance of $\beta$ is so small that it becomes negligible compared to all other errors.

The two effects can be summarised in the following equation, which is valid for $i<6$ :

$a_{i}\left(\phi_{\text {intra }}\right)=\alpha_{i}\left(a_{i}\left(\phi_{\text {cal }}\right)+\beta_{i}\right)$.

The pyramid provides spurious petal measurements under realistic operating conditions. This issue is further investigated in the following section, where we clarify the properties of $\alpha_{i}$ and $\beta_{i}$.

\subsubsection{Instabilities of the differential piston.}

We just showed the peculiar pyramid response to petal modes around static residual phases. We now study the way in which the AO loop dynamically accumulates errors on the petal modes in detail by observing their temporal evolution. The value of a petal mode coefficient $a_{i}$ taken alone does not show any specific information: $a_{i}$ has no meaning when it is not compared to its neighbours. Moreover, keeping the overall piston to be null over the entire pupil pulls all the petals in one direction when one of them is pushed in the other by any unexpected drift. As a consequence, the value of a single petal results as much from its own controlled value as from maintaining their global average $\frac{1}{6} \sum_{i<6} a_{i}$ to zero. 

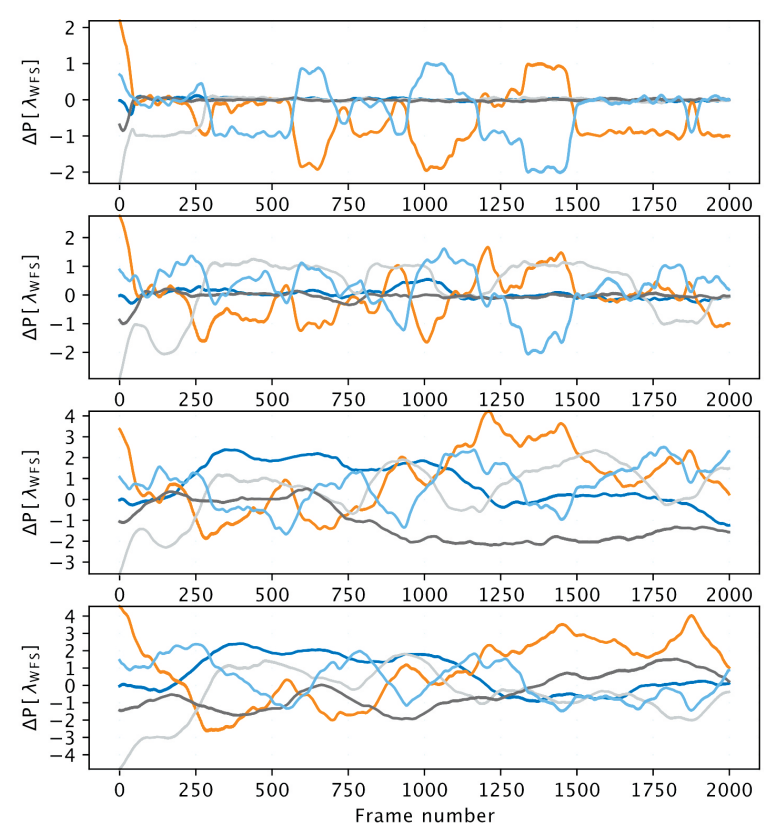

Fig. 6. Differential pistons fluctuations vs. AO frame number (frame 0 for loop closure and frame 2000 after $4 \mathrm{~s}$ ) with, from top to bottom, $r_{0}(500 \mathrm{~nm})=21.5,16.3,12.8$, and $8.9 \mathrm{~cm}$.

Conversely, from a physical standpoint, only the value of the difference between petals is meaningful and provides clues about how the pyramid and the AO loop are holding the petals in place. The relevant information is contained in the steps between neighboring petals, as these discontinuities across the spider are what triggers the pyramid signal related to petal modes. We define here the differential piston $\Delta P$ between petals as the difference between five petal modes and the first one, which we take as reference,

$\Delta P_{i}=a_{i}-a_{0}$.

In Fig. 6 we show the time evolution of $\Delta P$ fluctuations expressed in units of $\lambda_{\mathrm{WFS}}$ in four different seeing conditions (from top to bottom: $r_{0}(500 \mathrm{~nm})=21.5,16.3,12.8$ and $8.9 \mathrm{~cm}$ ) for a closed AO loop with the P-WFS and a loop frequency of $500 \mathrm{~Hz}$. There are five curves that are sometimes superimposed. For good seeing conditions, we observe that the $\Delta P$ values tend to stabilise around steps that are multiple of $\lambda_{\mathrm{WFS}}$, and as the $r_{0}$ decreases $(16.3 \mathrm{~cm})$, the transition rate between steps increases. At smaller $r_{0}(8.9 \mathrm{~cm})$, transitions become so frequent that we observe only random fluctuations of $\Delta P$ with large amplitudes, while the accumulation points around $\lambda_{\mathrm{WFS}}$ disappear.

We then face at least two issues. The first is the petal invisibility related to the interferometric phase wrapping of $\lambda_{\mathrm{WFS}}$. This fundamental physical effect cannot be avoided. A natural consequence of the periodicity of the petal response is to pull the control towards any integer multiple of waves, possibly away from the null optical path difference. The second is the invisibility of the petals due to the loss in sensitivity and the bias $\beta$ that is generated by the pyramid and is related to poor seeing and a low-Strehl regime.

While the problematic of $\lambda_{\text {WFS }}$ steps can be addressed using multi-wavelength sensing or a wavefront continuity hypothesis (see the discussions in Sect. 6), the source of random fluctuations and instabilities of the $\Delta P$ is the problem we study in the next section.

\section{Compensation of residual-induced sensor properties}

We here investigate the changes in the petal-mode sensing properties when in presence of large variance of $\mathrm{AO}$ residuals. We report difficulties in overcoming the sensitivity loss that is undergone by a $3 \frac{\lambda}{D}$ modulated P-WFS.

\subsection{Calibration-to-operation transfer matrix $\boldsymbol{T}$}

The interaction matrix obtained in operation in presence of AO loop residuals $\boldsymbol{D}_{\text {op }}$ differs from the calibrated one $\boldsymbol{D}_{\text {cal }}$. The reconstruction identity of Eq. (4) therefore no longer holds (Korkiakoski et al. 2007). In operation, we introduce a transfer matrix $\boldsymbol{T}$, defined as

$\boldsymbol{D}_{\text {cal }}^{\dagger} \boldsymbol{D}_{\text {op }}=\boldsymbol{T}$.

The calibrated reconstruction matrix $\boldsymbol{D}_{\text {cal }}^{\dagger}$ should then be updated to a modal reconstructor that is valid in operation $\boldsymbol{D}_{\mathrm{op}}^{\dagger}$, derived from a linear approximation, through the sole inversion of the diagonal of $\boldsymbol{T}$, denoted $\boldsymbol{T}_{\text {diag }}$,

$\boldsymbol{D}_{\text {op }}^{\dagger}=\boldsymbol{T}_{\text {diag }}^{-1} \boldsymbol{D}_{\mathrm{cal}}^{\dagger}$.

The diagonal elements of $\boldsymbol{T}_{\text {diag }}^{-1}$ are often referred to as modal optical gains (OG) in the literature. Different techniques have been proposed to retrieve this transfer matrix on sky and were validated for a continuous optical pupil (Deo et al. 2019; Chambouleyron et al. 2020). They assumed that $\boldsymbol{T}$ is a diagonally dominant matrix, with coefficients lying between 0 and 1 , as the consequence of a loss of modal sensitivity due to the presence of a non-null residual phase. The calibration-to-operation transfer matrix is also known to remain stable for a given seeing, that is, for an ensemble of residual phases that can be characterised with a common phase structure function $\mathscr{D}_{\phi}\left(r_{0}\right)$.

We here analyse results of simulations for an ELT case including the fragmented pupil and DM geometry. For various Fried parameters $r_{0}$, we compute interaction matrices $\boldsymbol{D}_{\mathrm{op}}$ around ten intra-petal uncorrelated residual wavefronts $\phi_{\text {intra }}$. The knowledge of the calibrated modal reconstructor $\boldsymbol{D}_{\mathrm{cal}}^{\dagger}$ enables us to derive a set of transfer matrices $\boldsymbol{T}$ using Eq. (12), which we use to characterise the modal sensitivity loss and the modal entanglement at each seeing.

\subsection{Sensitivity loss}

The diagonal coefficients of $\boldsymbol{T}$ provide the modal sensitivity loss coefficients $\left\{\alpha_{i}=t_{(i, i)}\right\}$ (Deo et al. 2019). Figure 7 shows these coefficients for all modes and at different seeing conditions. The sensitivity reduction encountered by the petal modes is located in the first five points of these curves. Compared to the $\phi_{i \geq 6}$, they undergo a drastic loss in sensitivity in presence of $\mathrm{AO}$ loop residuals, especially considering poor to median seeing conditions. The case of poor seeing conditions especially deserves attention as the petal-mode sensitivity drops to zero. The correcting factor to compensate for the modal sensitivity loss is usually computed as $1 / \alpha_{i}$. However, at $r_{0}(500 \mathrm{~nm})=8.9 \mathrm{~cm}$, the null sensitivity to the petals implies that it is impossible to mathematically retrieve some signal. Moreover, no temporal averaging of the slope measurements will help because no signal footprint will sum up.

We also note that the $\alpha_{i}$ coefficients vary from one petal to the next because there are preferred sensing directions depending on the alignment of the spider arms with respect to the four 


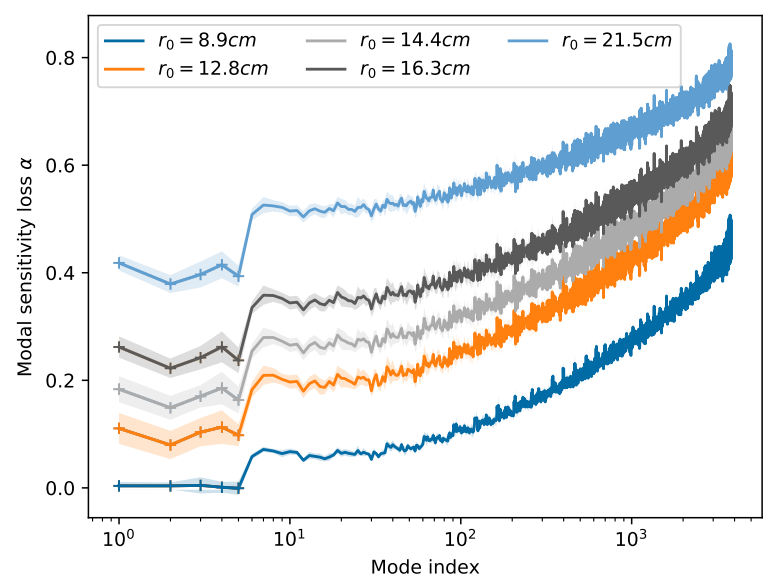

Fig. 7. Sensitivity loss coefficients $\alpha$ for seeing conditions ranging from $r_{0}=8.9 \mathrm{~cm}$ to $r_{0}=21.5 \mathrm{~cm}$. They are computed as an average of ten independent realisations of residual wavefronts with shaded areas of $\pm 1 \sigma$. The first five modes with cross markers denote the petal modes.

Table 2. Stability $\mu / \sigma$ of sensitivity loss coefficients for petal modes and intra-petal modes.

\begin{tabular}{lccccc}
\hline \hline$r_{0}$ at $500 \mathrm{~nm}(\mathrm{~cm})$ & 21.5 & 16.6 & 14.4 & 12.8 & 8.9 \\
\hline Petal modes & 12.5 & 7.0 & 4.8 & 2.8 & $\approx 0$ \\
Intra modes & 59 & 42 & 37 & 34 & 26 \\
\hline
\end{tabular}

edges of the pyramid and the sensor pixel matrix. These petal discrepancies would be evened out by applying a $\frac{\pi}{12}$ rad rotation of the telescope pupil and DM with respect to the P-WFS edges.

\subsection{Stability of compensation coefficients}

The reduction in sensitivity at a given turbulence amplitude is subject to large variations, which are seen in the $1 \sigma$ error bars in Fig. 7. We quantify the stability as the ratio between the mean value and the standard deviation of the $\alpha_{i}$ coefficients, computed over the ten considered residual phase realisations,

$\operatorname{stability}\left(\phi_{i}\right)=\frac{\mu\left(\alpha_{i}\right)}{\sigma\left(\alpha_{i}\right)}$.

Table 2 summarises the mean stability for petal and intrapetal modes at different seeing conditions. For example, for a 0.8 arcsec seeing, the stability of $\alpha_{i<6}$ is very poor, lower than 2.8. The sensitivity loss $\alpha_{i}$ does not only depend on the structure function of the residual phases $\mathscr{D}_{\phi}\left(r_{0}\right)$, but is also subject to stochastic fluctuations. Thus, the OG coefficients are always over- or underestimated: This leads to instabilities in the correction loop.

\subsection{Modal entanglement}

Figure 8 shows the coefficients of a line of $\boldsymbol{T}$ for a petal mode $\phi_{3}$ (top) and for an intra-petal mode $\phi_{100}$ (bottom) at a seeing of 0.8 arcsec. It highlights that the off-diagonal coefficients $t_{(3, j)}$ spread over all modes, with values of the same order of magnitude than the diagonal value $t_{(3,3)}$. This situation is drastically different for the 100th mode, where a clear diagonal coefficient appears distinctly from the others. As well as the sensitivity loss,
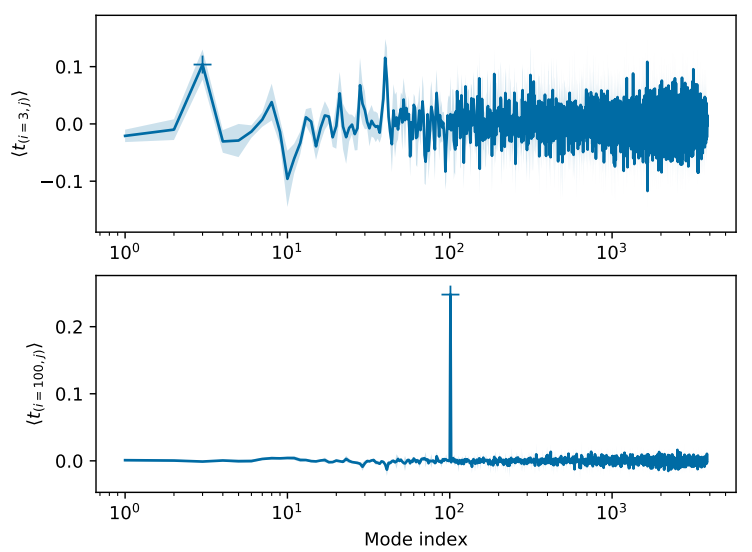

Fig. 8. Coefficients $t_{i, j}$ of a line $i$ of the operation-to-calibration transfer matrix $\boldsymbol{T}$ at a seeing of $r_{0}(500 \mathrm{~nm})=12.8 \mathrm{~cm}$. Top: for the third petal mode. Bottom: for an intra-petal mode with index 100 . The cross markers show the diagonal coefficients. Shaded areas indicate the $\pm 1 \sigma$ error bars.

$\alpha_{i}$ is given by the diagonal of $\boldsymbol{T}$, we introduce $c_{i}$ coefficients to characterise the strength of the cross-talk between modes when operating on-sky and defined by the off diagonal coefficients of the transfer matrix. For mode $i$, we have

$c_{i}=\sqrt{\sum_{j \neq i}\left|\frac{t_{i, j}}{t_{i, i}}\right|^{2}}$.

The $c_{i}$ coefficients are shown in Fig. 9. The behaviour difference between the coefficients of the petal modes and those of the other modes is striking. They are about ten times larger for the petal modes than the others. The cross-talk strength increases with the residual amplitude: the smaller $r_{0}$, the denser the transfer matrix $\boldsymbol{T}$, and the larger the P-WFS non-linearity.

In Sect. 3 we introduced the concept of a bias $\beta$ found in the estimated phase and reflecting the fictitious $\Delta P$ measurement introduced by the P-WFS, even when no petal residuals are present in the incoming phase. This bias originates from the presence of modal cross-talk between petal modes and the AO loop residuals at the location of the $\Delta P$ signature. The latter is weak and concentrated in the shaded area below the spider arms, which means that it is encoded with only a few pixels and is easily corrupted by other signals. We note that we here only account for the modal cross-talk belonging to the DM frequencies, but the higher frequencies of the fitting error term also contribute to $\beta$. Hence, we cannot retrieve the true generated bias by only analysing the P-WFS modal response: the knowledge of this bias $\beta$ relies on the knowledge of the of-diagonal coefficients of $\boldsymbol{T}$, weighted by the spectra of the modal residuals, and on the bias induced by the fitting error term. As the of-diagonal coefficients of $\boldsymbol{T}$ are approximately uniform at all frequencies, and as the spatial power spectra of the residuals are mainly composed of low-order aberrations, we expect the latter to have more weight in this phenomenon. Finally, we add that we encountered the same instabilities as with the diagonal elements when we considered the of-diagonal coefficients of $\boldsymbol{T}$ from one residual phase to the next.

\subsection{Conclusions for the use of optical gain compensation in a fragmented-pupil geometry}

To overcome sensitivity reduction, the use of scalar-based, OG compensation techniques is already widely studied. However, 


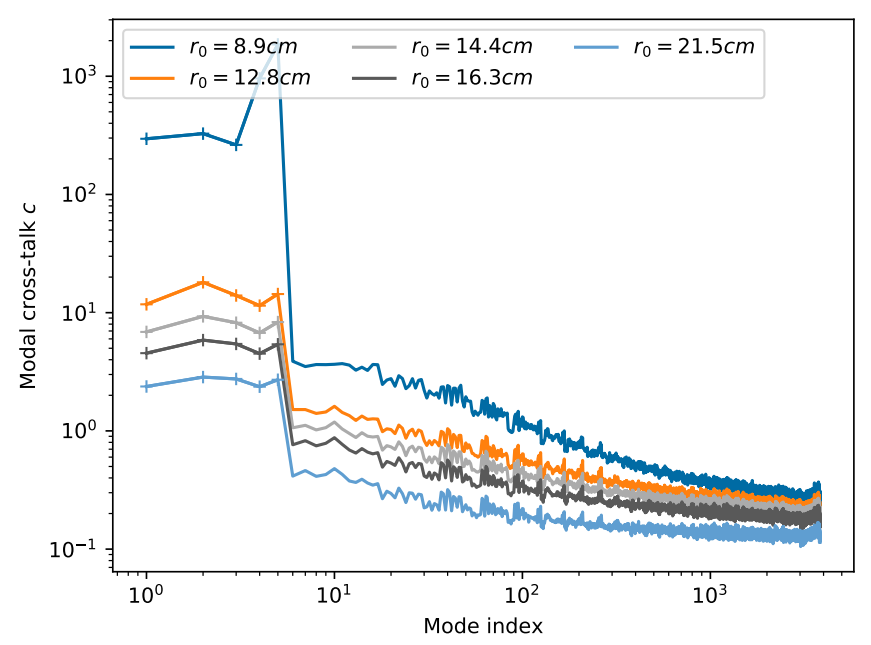

Fig. 9. Modal cross-coupling coefficients $c_{i}$ for seeing conditions ranging from $r_{0}(500 \mathrm{~nm})=8.9 \mathrm{~cm}$ to $21.5 \mathrm{~cm}$. They are computed as an average of ten independent realisations of residual wavefronts. The first five modes with cross markers denote the petal modes.

we highlight that fragmented pupils deserve a special amendment to these frameworks. For the petal modes, we show that the sensitivity is dramatically reduced, by up to about $10^{-3}$ for poor seeing conditions (seeing $>1$ arcsec). Petals belong to the invisible modes space of the P-WFS, and their measurement is completely dominated by the cross talk between petals and intra-petal residuals. For median to good seeing conditions (seeing $<1$ arcsec), the sensitivity to petals can be compensated with modal gain compensation. However, this compensation will suffer from significant under- and overestimation because part of the sensitivity loss is driven by large stochastic fluctuations and does not only depend on the structure function of the residual phases $\mathscr{D}_{\phi}\left(r_{0}\right)$. Finally, the transfer matrix $\boldsymbol{T}$ is dense: for petal modes, the of-diagonal coefficients are not negligible. This modal cross-talk impacts the measurement and introduces a bias $\beta$. At a given seeing, this bias follows a normal distribution with null expected value. The poorer the seeing, the larger the variance of $\beta$ : This error will mainly affect seeing conditions where the P-WFS is already little sensitive to petal modes. The erroneous measurement due to $\beta$ adds up to the phase-wrapping petal invisibility, and in these conditions, the petal modes can become out of control.

Altogether, this makes the OG compensation for petal modes a critical but likely impossible issue. The constraints we described that are specific to $\Delta P$ do not hold within the hypothesis underlying the current proposed solutions for tracking PWFS operating changes (Chambouleyron et al. 2020; Deo et al. 2021). First, the density of $\boldsymbol{T}$ is always neglected by the OG trackers, which simply proceed to a linear scaling of the modal reconstruction. Then, the instabilities of the diagonal coefficients will not be accurately depicted. Finally, the measured OG to be applied to petals are infinite in harsh atmospheric conditions, meaning that the transfer matrix $\boldsymbol{T}$ is ill-conditioned. Because of this ill-conditioning, we note that a "good" modal basis for AO control should not explicitly contain petal modes. This issue will, however, feed through to the gains that are applied to the modes on which the petals are projected (basically all the modes and especially the lower ones, where the use of OG compensation is the most critical). Because we considered only five modes with respect to about 4000 modes, however, the non-orthogonality of $\boldsymbol{T}$ in presence of large AO residuals is weak.

\section{Sensitivity-enhancement strategies}

In this section, we explore different strategies to increase the $\Delta P$ sensitivity with the P-WFS, first using OG compensation to minimise the intra-petal residuals, then using different modulation paths, and finally, we investigate the possibility of increasing the number of edges of the pyramid. We show closed-loop results with a wide range of $\frac{D}{r_{0}}$ such that any instrument at the ELT, from visible to infrared P-WFS, may relate to the results we present.

\subsection{Simulation details of the seeing conditions}

We operated simulations with atmospheric conditions ranging from $7.6 \mathrm{~cm}$ to $76 \mathrm{~cm}$ Fried parameter expressed at $500 \mathrm{~nm}$, noted $r_{0}(500 \mathrm{~nm})$. The simulations were performed with a sensor working at a visible sensing wavelength $\lambda_{\mathrm{WFS}}=700 \mathrm{~nm}$. The $D / r_{0}\left(\lambda_{\mathrm{WFS}}\right)$ is just noted $D / r_{0}$ for the sake of simplicity when expressed at this wavelength. These paramaters map to a $D / r_{0}$ of 35-350 considering the ELT pupil diameter. Any ELT AO system can be related to our graphs using the $r_{0}$ value expressed at the sensor wavelength: For a typical $r_{0}(500 \mathrm{~nm})=12.8 \mathrm{~cm}$, we have $D / r_{0}=200$ in the visible band (MICADO and HARMONI cases $)$ or $D / r_{0}(2200 \mathrm{~nm})=50$ with a sensor in $K$ band, such as for METIS. We obtained a statistical average by running five independent simulations with a length of 4 seconds: each of the five phase screens was a single atmospheric layer generated with a different seed of the random number generator. They evolved with a wind speed of $10 \mathrm{~m} \cdot \mathrm{s}^{-1}$, which renewed the phase screens within the telescope pupil every 4 seconds. To properly take the high spatial frequencies of the atmospheric turbulence into account, we sampled them with at least 3 pixels per $r_{0}$. This severely impacts the simulation speed for poor seeings: A simulated iteration with a $3 \frac{\lambda}{D}$ modulated PSF would typically run at $16 \mathrm{~Hz}$ for median $D / r_{0}$ values, compared with $4 \mathrm{~Hz}$ for the largest ones. This effort is mandatory, however, if meaningful results are expected.

The varying parameters we used in the different simulations are summarised in Table 3, where the use of "CLOSE" and "clover" is developed in the following sections.

\subsection{Closed-loop performance criteria}

In this section, we distinguish the petal-error contribution from the intra-petal residuals in the global performance. To do this, the closed-loop residual phases were projected onto $B_{\text {petal }}$, giving the $a_{i}$ coefficients of the phase as defined in Eq. (5) and giving the possibility of defining two performance criteria.

First, we assessed the ability of the pyramid to sense petal modes regardless of the $\lambda_{\mathrm{WFS}}$ wrapping, which is a physical limitation inherent to any sensor. As stated in Sect. 3, the value of a petal taken alone has no physical meaning, but their differences with one another is relevant and is tied to the imaging quality. We defined a criterion for the petal error that relies on the expression of the SR expressed at the sensing wavelength as it provides results that are independent from the modulo $\lambda_{\mathrm{WFS}}$ wrapping. The effect of piston errors has been subject to numerous studies in the context of telescope segmentation and multiaperture interferometry. The analytical expression of the SR for 
Table 3. Summary of the simulation settings.

\begin{tabular}{lcccc}
\hline \hline Gain method & Modulation & Faces & Figures & comments \\
\hline$g=1$ & $3 \frac{\lambda}{D}$ & 4 & 10 & Local wavefront divergence $^{(a)}$ \\
CLOSE & $3 \frac{\lambda}{D}$ & 4 & $10,12,14$ & - \\
CLOSE & $1 \frac{\lambda}{D}$ & 4 & 12 & Local wavefront divergence $^{(a)}$ \\
CLOSE & Clover & 4 & 12 & - \\
CLOSE & $3 \frac{\lambda}{D}$ & 6 & 14 & - \\
\hline
\end{tabular}

Notes. ${ }^{(a)}$ Local wavefront divergence stands for the divergence of actuators located at the edge of the pupil occurring at $D / r_{0}\left(\lambda_{\mathrm{WFS}}\right)=350$.

a fragmented pupil is derived from Chanan \& Troy (1999) and Yaitskova et al. (2003). We applied it for a six-petal geometry,

$\mathrm{SR}_{\mathrm{P}}=\frac{1}{36} \sum_{i<6} \sum_{j<6} \cos \left(a_{i}-a_{j}\right)$.

We then defined the variance of the petal error as

$\sigma_{\mathrm{P}}^{2}=\left\langle-\ln \left(\mathrm{SR}_{\mathrm{P}}\right)\right\rangle$.

Secondly, we are also interested in the intra-petal residual wavefront, quantified with $\sigma_{\text {intra }}$. It is defined as the quadratic sum of the $a_{i}$ coefficients related to the projection of the residual phase $\phi_{\text {op }}$ onto the intra-petal modes $\phi_{i>6}$, plus a fitting term,

$\sigma_{\text {intra }}^{2}=\sum_{i \geq 6}\left\langle a_{i}^{2}\right\rangle+\sigma_{\text {fitting }}^{2}$.

We computed $\sigma_{\text {intra }}^{2}$ and $\sigma_{\mathrm{P}}^{2}$ from the residual phase at each iteration, and we took the temporal average over the total length of the simulation expressed with the symbol $\langle\ldots\rangle$. The data acquisition starts 100 iterations after loop closure to ensure loop convergence. Finally, we took the square root of these terms in order to express them in radians at the wavelength of the WFS.

\subsection{Use of optical gain compensation}

\subsubsection{Method}

We first investigated as a basic starting point the petal and intrapetal errors using a loop integrator gain of 1 . This setting is possible because the natural loss in sensitivity of a visible PWFS on median conditions on the ELT is in the range 0.2 to 0.5 (Deo et al. 2019): This sets the overall gain of the loop to a value that is not optimal, but that is stable and acceptable for turbulence rejection.

In a second step, we used the OG compensation algorithm CLOSE from Deo et al. (2021), in order to keep the system optimised against the modal sensitivity loss. The principle of CLOSE is to use the temporal auto-correlation of the pyramid modal response to provide a real-time tracking of modal integrator gains at any varying observing conditions. The setting of the hyper-parameters was chosen empirically to deliver the best results for a seeing of 1 arcsec in the ELT case. Although parameters were not specifically optimised for each atmospheric conditions we address, we were able to verify that the results were satisfactory for all simulations. We chose a null set-point and learning factors $\left(q^{+}=1 / 200 ; q^{-}=1 / 100\right)$ : The modal gains can decrease faster than they may grow, which is beneficial for the robustness of the loop.

In both cases of unitary gain and CLOSE algorithm, we used the full modal reconstructor $\boldsymbol{D}^{\dagger}$.

\subsubsection{Results}

We show in Fig. 10 the petal and intra-petal errors at different $D / r_{0}$ using a unitary scalar integrator gain $g=1$, in blue, and using CLOSE to perform OG tracking, in orange. We add the ideal aliasing and fitting error computed for a triangular actuator geometry that would be obtained for a perfect linear AO system as the dashed black line.

The impact of OG compensation is especially prominent in poor seeing conditions. By partially reducing the pyramid nonlinearity, it allows improving the $\sigma_{\text {intra }}$ error by a factor of 1.5 for $D / r_{0}=350$, together with reducing the statistical dispersion of the performance. As a side effect, this PSF improvement is beneficial for the measurement of the differential pistons as well: the decrease in $\sigma_{\text {intra }}$ has a slight beneficial effect by reducing $\sigma_{\mathrm{P}}$. However, this is clearly not sufficient to set the pyramid in a regime where it is reliably sensitive to $\Delta P$. For $D / r_{0}>300$, the $\sigma_{\mathrm{P}}$ error saturates at a value of $1.5 \mathrm{rad}$, which corresponds to completely random temporal fluctuations of the petal modes. This regime is reached as soon as the intra-petal error exceeds $2 \mathrm{rad} \mathrm{rms}$. With a four-face pyramid modulated at $3 \frac{\lambda}{D}$, the use of OG does not help significantly to retrieve the petal sensitivity. Even worse, at strong seeings, we verified that as the petal modes are unseen by the sensor, CLOSE or any other linearbased OG compensation algorithm boosts the presence of the unseen modes in the correction loop and is detrimental for the AO correction loop. This is characterised by even larger amplitudes of the $\Delta P$ fluctuations. This effect is not seen in Fig. 10 because the performance metric is taken after a $\lambda_{\mathrm{WFS}}$ wrap; but for a seeing of 0.8 arcsec, the amplitude of the $\Delta P$ fluctuations is about 1.5 times larger with the use of CLOSE than with a unitary integrator gain (see Fig. 6). A way to reduce this amplitude is to use automatic OG compensation techniques only for intra-petal modes and using a constant gain for petal modes.

\subsection{Increase in time spent on pyramid edges: Modulation path}

As described in Sect. 3, the pyramid is sensitive to the differential piston mostly where the incoming beam passes at a distance smaller than $1 \frac{\lambda}{D}$ from the pyramid edges (see Fig. 3). We call this region the diffractive area. To increase the time spent on it, we study the impact of two parameters: the beam path generated by the modulating mirror studied in this section, and then the number of faces of the pyramid, studied in Sect. 5.5.

\subsubsection{Method}

The pyramid is usually operated with a circular and uniform modulation, which we previously introduced using a modulation radius of $r_{\text {mod }}=3 \frac{\lambda}{D}$. The modulation is known to improve the trade-off between sensitivity and linearity, especially for 

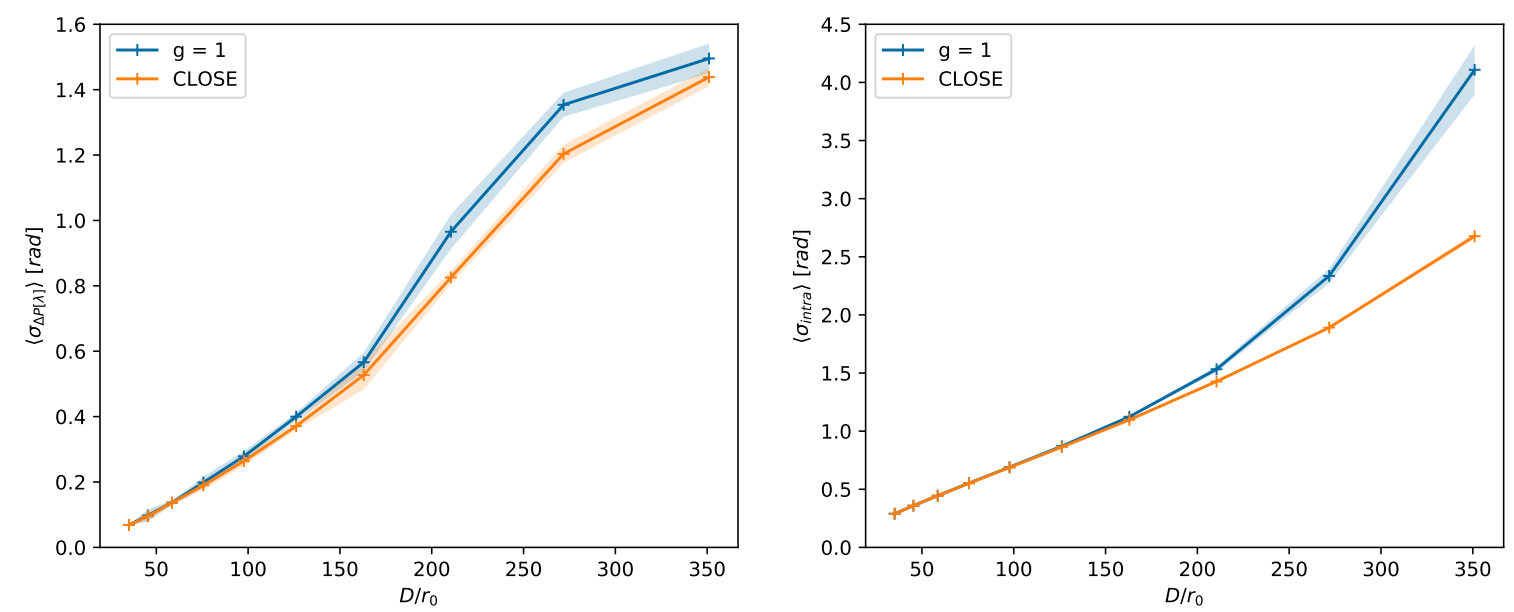

Fig. 10. Petal (left) and intra-petal (right) standard deviations expressed in radians without modal gain compensation (integrator gain set to 1, in blue) and with the use of CLOSE algorithm (null setpoint and learning factor $\left(q^{+}=1 / 200, q^{-}=1 / 100\right)$, in orange). The component of fitting and aliasing errors for a triangular actuator grid is shown as the dashed grey line. Shaded areas: $\pm 1 \sigma$ error bars.
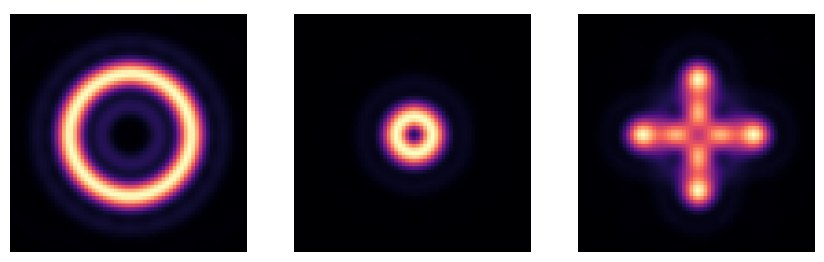

Fig. 11. Pyramid modulation paths: Left: $3 \frac{\lambda}{D}$ circular modulation. Middle: $1 \frac{\lambda}{D}$ circular modulation. Right: clover modulation using hypotrochoid equations with $q=4, k=2$ and a modulation "radius" $r_{\bmod }=3 \frac{\lambda}{D}$.

low-order modes. Engler et al. (2019) showed that an unmodulated P-WFS is effective in reducing the differential pistons. However, poor seeing conditions and high $\mathrm{AO}$ residuals require increasing the dynamic range of the sensor, hence the modulation radius, which is inimical to petal-mode sensing. The circular modulation therefore does not offer a trade-off that fully suits our needs.

A cloverleaf-shaped modulation path aims at spending more time close to the diffractive area while keeping a modulation amplitude of $\pm 3 \frac{\lambda}{D}$ for an improved dynamic range. These are hypotrochoids, that is, plane curves defined as the sum of two circular motions in opposite directions. They allow a large variety of modulation paths, and their parametric equations are given by the sum of two sines with different harmonics,

$\left\{\begin{array}{l}x(\theta)=\frac{r_{\text {mod }}}{q-1+k}((q-1) \cos \theta+k \cos ((q-1) \theta)) \\ y(\theta)=\frac{r_{\text {mod }}}{q-1+k}((q-1) \sin \theta-k \sin ((q-1) \theta))\end{array}\right.$.

The parameter $r_{\text {mod }}$ stands for the modulation radius when circular modulations are considered, and we used this same term to express the largest excursion from the centre of the hypotrochoidal pattern. The term $q$ drives the number of branches of the hypotrochoid and is here set to 4 to match with the number of pyramid faces. We note that this term imposes that the tip-tilt mirror oscillates at a higher frequency than usual. Finally, $k$ is a free parameter that for $k=0$ corresponds to a circular modulation and for $k=q-1$ corresponds to a modulation path that crosses the tip of the pyramid.

Figure 11 shows three modulation paths we investigated here: Two circular modulations with $3 \lambda / D$ and $1 \lambda / D$ modula- tion radius and a clover using $k=2$, which does not exactly cross the tip of the pyramid but lengthens the time spent on the edges. Two modulation points are separated with the same temporal gap. The brighter areas of Fig. 11 underpin that the clover modulation slows down when the beam crosses the edges farther from the centre, which is exactly our aim.

For all simulation results presented in this section, we used CLOSE to compute the modal integrator gains in order to partly compensate for the P-WFS non-linearity. This is especially needed when using small modulations because higher optical gain effects are observed.

\subsubsection{Results}

As shown in Fig. 12, reducing the modulation radius enables us to increase the sensitivity to petal modes and lower $\sigma_{\mathrm{P}}$. However, this solution is not viable for harsh atmospheric conditions when a good linearity range is needed to overcome the large turbulent phase amplitudes. Despite the use of modal gains, the circular modulation of $r_{\text {mod }}=1 \lambda / D$ comes with a deterioration of the intra-petal performance at large $D / r_{0}$, followed by a poorer correction of petals. Using a clover modulation allows us to find a finer compromise in terms of sensitivity and dynamic range. The results are especially promising for good seeing or large sensing wavelengths where the $\sigma_{\mathrm{P}}$ error stays below $\frac{\pi}{10}$ (we recall that we did not consider here the $\lambda_{\mathrm{WFS}}$ steps of the petals). Nevertheless, we observe stronger non-linear effects at large $D / r_{0}$ : This can be explained, because the dynamic range of the clover modulation is found in only two directions, along by the pyramid edges, while the beam path amplitude is on average smaller than for a circular modulation of $3 \frac{\lambda}{D}$. The gain in the $\Delta P$ sensitivity with the clover is not sufficient to overcome the impact of the increase in $\sigma_{\text {intra }}$ residuals for $D / r_{0}=350$.

To overcome this intra-petal performance loss, we could imagine increasing the value of $q$, that is, improving the dynamic range in a larger number of directions (leaves of the clover), thus having a larger average modulation, but spending more time out of the diffractive area, which is counterproductive to the intended purpose. We could also increase the amplitude $r_{\text {mod }}$ of the clover path and adjust the factor $k$ to keep the beam as close as possible to the edges.

In the end, the use of clover modulation is an interesting solution for instance for a P-WFS in $K$ band and even in the case of 

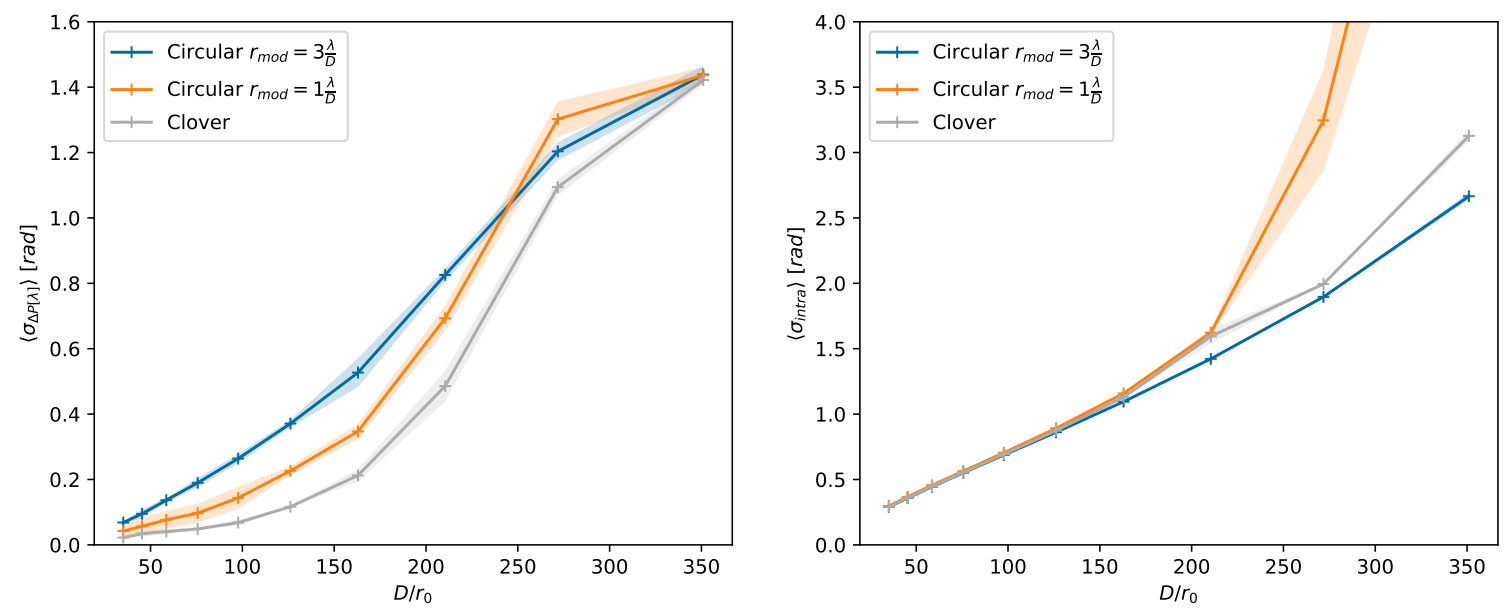

Fig. 12. Petal (left) and intra-petal (right) standard deviations expressed in radians for different modulation paths of the tip-tilt smearing mirror: Two circular modulations with a modulation radius of $3 \frac{\lambda}{D}$ (blue) and $1 \frac{\lambda}{D}$ (orange) and a clover modulation with an amplitude of $3 \frac{\lambda}{D}$ and a parameter $k=2$. We use the CLOSE algorithm to compensate for the P-WFS non-linearity (null setpoint and $\left(q^{+}=1 / 200, q^{-}=1 / 100\right)$ ). Shaded areas: $\pm 1 \sigma$ error bars.
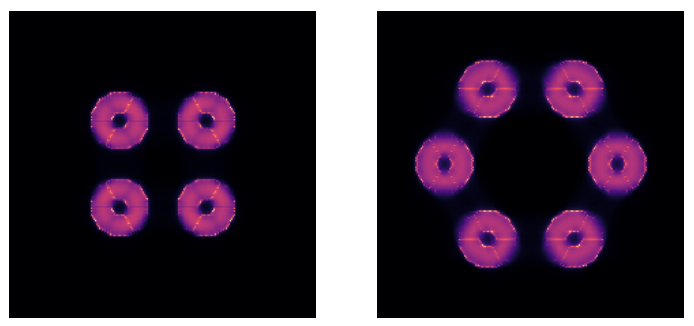

Fig. 13. Pyramid detector plane using a four-face pyramid mask (left) and a six-face pyramid mask (right). The distance between the pupil centres is 72 pixels.

good seeing in the visible; but for stronger seeings, it will remain difficult to find an efficient trade-off between petal and intra-petal sensitivity.

\subsection{Increase in time spent on pyramid edges: Number of faces}

\subsubsection{Method}

Increasing the number of faces enables us to add diffracting parts of the pyramid. It improves the sensitivity to petal modes, but splitting the beam into a higher number of pupils comes with a decrease in intra-petal mode sensitivity, which we once again aim to compensate for using modal gains (in addition to the fundamentally increased noise for the faintest guide stars). We study here a six-face pyramid with edge directions chosen to maximise the petal-mode sensitivity. Unlike the four-face pyramid, it does not have any preferred direction to sense petal modes. The number of slopes is increased by a factor 1.5 and would slow-down real-time the modal reconstruction computation (Fig. 13).

\subsubsection{Results}

Figure 14 shows that as expected, the use of a six-face pyramid clearly improves the measurement of $\Delta P$ compared to the case of four faces. It allows us to keep a reliable measurement for $D / r_{0}<200$, that is, when the curves of the intra-petal residuals of the four- and six-face pyramid are superimposed. For poor seeings, we can realise how thin the boundary between petal modes and intra-petal mode sensing is. A slight increase in $\sigma_{\text {intra }}$, explained by the poorer sensitivity to intra-petal modes that the OG fails to fully compensate for, and the benefit of the six-face pyramid is forfeit. In the end, the performance obtained with the six-face pyramid is quite comparable to the one studied with the clover modulation. However, this method requires a new hardware design and increases the number of pyramid measurements, here by a factor 1.5 . Hence, it impacts the RTC computing requirements, as well as readout and photon noises, in an unfavourably way compared to the small improvement that is obtained in return.

Finally, considering the simulations carried out in Sects. 5.3-5.5, we derive an empirical boundary on the intra-petal residuals of about $\pi / 2$ rad at $\lambda_{\mathrm{WFS}}$. Beyond this limit, the measurement of petal modes with the pyramid becomes an impossible task.

\section{Discussions}

In the framework of this paper, we conducted numerous simulations with the intention to follow the true ELT geometry as closely as possible (pupil geometry, fragmented DM, null interactuator coupling, etc.). In this section we therefore provide comments and returns on the parameters we experimented with to successfully close the AO loop, especially in presence of large wavefront amplitudes. We also discuss a list of parameters we did not emulate, but that will impact the P-WFS sensing regime, the AO loop control quality, and thus, by feedback, the petalmode measurement quality.

\subsection{Feedback on closing an AO loop at the ELT scale}

Here we present three key parameters that we have identified as significantly impacting our results while they could appear as secondary parameters at first sight. We detail here the effects we observed depending on the chosen strategy.

Actuator selection. The first step we performed was a selection of the valid actuators (see Appendix A), chosen as degrees of freedom to perform the computation of a modal basis. This was done using a criterion on their presence within or absence from a given annulus defined with two radii. We performed this 

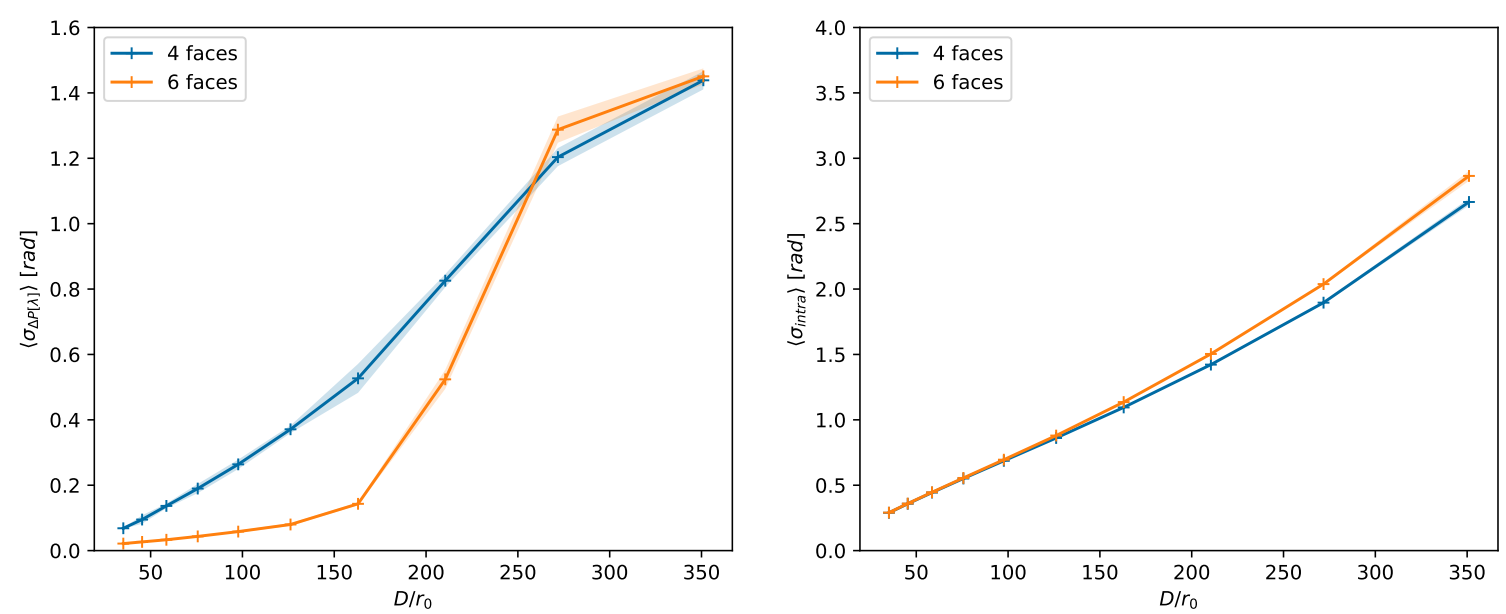

Fig. 14. Petal (left) and intra-petal (right) standard deviations expressed in radians for a four-face and a six-face pyramid. We use the CLOSE algorithm to compensate for the P-WFS non-linearity. Shaded areas: $\pm 1 \sigma$ error bars.

selection such that 3852 actuators were controlled as degrees of freedom, and we extended the modes to the remaining passive actuators. When using a wider selection, some of the actuators located close to the pupil edges were facing divergence, as these were poorly seen by the sensor. This behaviour, moreover, is similar to the petal-mode divergence we studied here: The poor sensitivity of actuators deteriorates even more in the presence of large AO residuals and leads to actuators that are out of control. As an actuator diverges, the local tilt in the wavefront shape implies that none of the four P-WFS sub-pupils encoding the actuator phase information are illuminated. Ultimately, this causes the divergence to be unrecoverable by the sole use of the sensor.

In this respect, the selection of the active actuators is critical. The extension of the modal basis using a Kolmogorov hypothesis through an MMSE operator was found to be a good approach to perform the correction over the full DM space while keeping the reconstruction to a smaller number of actuators (see Appendix C).

Number of controlled modes. If it is necessary to limit the number of actuators, the number of controlled modes is also a decisive criterion to optimize AO loop correction. To deal with strong seeings, a first approach was to filter a few hundred modes with the idea to keep a stable modal correction. The effect was counterproductive. By giving up on the compensation of high spatial frequencies, the AO residual amplitudes increased and completely altered the pyramid operating regime, starting obviously with a divergence of the petal modes. This led to a loop divergence that could not be avoided using different modal gain optimisation strategies. This means that the presence of poorly seen modes or actuators in the command space is critical to the efficiency of the correction, and we strongly recommend that these modes are identified and filtered out; we recommend explicit identification of blind modes rather than truncation of high frequencies. We also note that truncation or regularisation from the singular values of $\mathbf{D}_{\text {cal }}$ should be performed carefully because in the case of normalised modes, low-order modes have the weakest sensitivity and will be truncated first.

Number of sub-apertures. The number of pyramid subapertures along the pupil diameter has two consequences for the AO loop. We performed simulations with 87 and 95 subapertures in the pupil diameter, that is, a ratio of $4 / 3$ against $3 / 2$ with respect to the number of actuators in the pupil diameter.
First, by having a better sampling of the modal response, the intra-petal error is reduced. Second, more pixels are available to encode the petal modes signature. This makes the system more robust, at least for the noise-free simulations we performed.

\subsection{Other sources of petal-mode mismeasurement}

We conducted this study focusing only on the seeing conditions, but we identified other observation parameters that will affect the pyramid operating regime. The expected reliability of the onsky measurement of the modes that are already poorly seen in an ideal simulation is a question to be raised. The proper petal-mode correction often comes down to very little. We here propose a non-exhaustive list of issues that might cause an amplification of the errors. This list remains to be studied and to be considered in the design of the AO controller.

- The simulations we performed were made without taking into account any contribution of the noise. However, at low flux, photon noise and detector readout noise -if any- will deteriorate the signal-to-noise ratio. This decrease comes with an increase of the residual amplitude despite the use of OG compensation. For instance, a simulation run with photon noise on a guide star magnitude $M_{R}=16$ with a 0.8 arcsec seeing, that is, $D / r_{0} \approx 200$, places the pyramid working in visible band in a regime in which it is insensitive to petal modes with $\sigma_{P}=1.5 \mathrm{rad}$.

- The non-common path aberrations (NCPA) correction modifies the P-WFS response. The gain-tracking approach aims to change the sensor reference after subtraction of the NCPA wavefront. Although NCPA can be calibrated and is expected to be a quasi-static low-order aberration, therefore being well known, the possibility of non-linear effects of their subtraction on poorly sensed modes should be studied (Chambouleyron et al. 2021).

- The mechanical structure of the telescope will undergo dynamic perturbations leading to misalignments between the pupil footprint and the DM. Moreover, the DM itself will be subject to displacements of the actuator grid with respect to the WFS sup-apertures, called misregistrations (Heritier et al. 2018).

- The final alignment of the primary mirror segments will be limited by the residual piston, tip, and tilt (phasing errors), and the low-order surface figure errors (segment shape). 
The instruments equipped with P-WFSs will likely detect some of the residual quasi-static aberrations of the telescope and correct them with the DM: Phasing will be within the AO system spatial bandwidth, whereas segment shapes and edges will be barely sampled by the instrument sensors and will cause some aliasing in the modal response. Aliasing will be mitigated by P-WFS over-sampling, but the final impact remains poorly known.

\subsection{Dealing with the $\lambda$ ambiguity}

In this paper, we deliberately omitted the problem of the $\lambda_{\mathrm{WFS}}$ wrapping that is encountered by any interferometric sensor when $\Delta P$ is measured. To compensate for turbulence-induced petal modes, continuity constraints on the WF reconstruction have shown promising results, such as with the pairing method (Schwartz et al. 2018) or the continuous-basis approach (Bertrou-Cantou et al. 2020). However, these methods will fail to compensate for telescope-induced discontinuities in the incoming wavefront (LWE, thermo-mechanical drift of the DM, etc.), for which a dedicated optical device is necessary. We can expect the LWE to occur during good observation nights, when the wind is too weak to homogenise the air temperature across the pupil. The observing conditions at small $D / r_{0}$ are conducive to petal-mode measurement. However, the sensor will still suffer from its limited capture range. To increase the capture range, we can either use the two-wavelength approach (Pinna et al. 2006), or we have to shift the sensor to a higher wavelength. Plus, we have shown that the latter is also in favour of petal sensitivity. The design of a sensor specifically targeting the phase steps across the spiders will fully depend on the disturbances induced by the telescope thermo-mechanical behaviour.

\section{Conclusions and perspectives}

The ELT spider arm width requires an adaptive phasing of the petals of which its optical pupil is composed. The pyramid wavefront sensor was selected for its great sensitivity and adaptive linearity range, but it exhibits a highly variable sensitivity to the phase steps across the telescope spider arms depending on the observing conditions. The paper highlights a measurement issue of the petal modes that is caused by two main reasons. First, petal modes suffer from a sensitivity loss to a much larger extent than the other modes do when in the presence of AO residual WF errors. This sensitivity even drops to zero considering strong seeing for a P-WFS in the visible, when the PSF filtered by the pyramid mask is not tight enough to let the petal-mode signal out, the latter being substantially contained in the central core of the PSF (or the breaking thereof into several lobes in the presence of LWE). Secondly, the optical signature of petal modes in the sensor pupil is located in a narrow region along the spider arms, and it is rapidly drowned among the wavefront residuals. Cross-talk occurs between the petal signal and the intra-petal signal and leads to a biased pyramid measurement.

The optical gain compensation strategies hitherto relied on the hypothesis of a stable, diagonally dominant and wellconditioned calibration-to-operation transfer matrix. We highlight that this hypothesis no longer holds for petal modes. The stability of the applied gain is dominated by stochastic fluctuations and does not only depend on the Fried parameter; plus, a modal coupling is induced by the presence of both the DM fitting error and the incomplete residual loop compensation of mirror modes (loop temporal error, non-linearity, etc). The illconditioning of the OG compensation occurs with strong seeing, when the sensitivity to the petal modes is reduced to zero.
The presence of these five unseen modes in the control space together with the regular OG tracker steps up the amplitude of the differential piston fluctuations and causes an amplification of the injected errors by the AO controller. This comment equally applies to any poorly sensed mode or actuator.

From the steering mirror modulation radius to the number of sub-apertures in the sensor pupil, the actual design of the ELT instrument SCAO systems was driven by the optimisation of the intra-petal performance. We performed extensive simulations using a proper ELT DM model and adding the petal-mode fluctuations to the error budget to investigate new designs for the P-WFS. We explored techniques that lengthen the time spent by the PSF core on the diffractive region of the sensor, either using an appropriate modulation or adding more edges to the pyramid phase mask.

These solutions yield promising results for seeing conditions up to $D / r_{0}<200$, corresponding to intra-petal errors typically up to $\pi / 2 \mathrm{rad}$ rms at $\lambda_{\mathrm{WFS}}$. Under these conditions, they effectively allow the system to decrease the petal error while maintaining the intra-petal performance. Instruments such as METIS, using a near-infrared pyramid sensor, can benefit from these methods. Conversely, at larger $D / r_{0}>200$, these strategies fail to provide reliable results. The balance between the petal and intra-petal sensitivity is more difficult to reach for visible SCAO systems (HARMONI and MICADO). The petal and intra-petal error budget terms are closely related, but they follow an opposite pyramid-sensing logic: The gain in terms of $\Delta P$ sensitivity is paid for with a loss in sensitivity or dynamic range of the intrapetal modes, which increasing error turns against the petal-mode sensitivity: there is no way out.

Throughout this article, we set aside the problem of the $\lambda_{\text {WFS }}$ ambiguity that underlies the wave property of light. From a controller point of view, it can be tackled using the wavefrontcontinuity hypothesis. However, phase-step fluctuations across the spiders can appear in addition to the atmospheric induced $\Delta P$ (e.g. low-wind effect; Milli et al. 2018) and will induce discontinuities outside the wavefront-continuity hypothesis framework. For P-WFSs in the visible, two limitations arise: The sensor capture range is short, and these errors will not be measured because of the presence of AO loop residuals. These conclusions advocate for an additional sensor specifically targeting the petalmode measurement issue. One of all the various possible choices might be a P-WFS working at near-infrared wavelengths. The implementation of such a device will be driven by the spatial and temporal properties of the phase discontinuities.

Acknowledgements. We especially want to thank Henri Bonnet for many insightful comments and fruitful discussions around the petalling effects and for providing us with high quality, reliable, properly sampled data of the influence functions of the M4 deformable mirror. This research is performed in the frame of the development of MICADO, first light instrument of the ELT (ESO), with the support of ESO, INSU/CNRS and Observatoire de Paris. This work was partially supported by the WOLF project (ANR-18-CE31-0018) of the French National Research Agency (ANR). V. D. acknowledges support from NASA grant \#80NSSC19K0336.

\section{References}

Bertrou-Cantou, A., Gendron, E., Rousset, G., et al. 2019, in 6th AO4ELT conference-Adaptive Optics for Extremely Large Telescopes

Bertrou-Cantou, A., Gendron, E., Rousset, G., et al. 2020, Proc. SPIE, 11448, 1144812

Brandl, B. R., Absil, O., Agócs, T., et al. 2018, in Ground-based and Airborne Instrumentation for Astronomy VII, eds. C. J. Evans, L. Simard, H. Takami, et al., SPIE Conf. Ser., 10702, $107021 \mathrm{U}$

Burvall, A., Daly, E., Chamot, S. R., \& Dainty, C. 2006, Opt. Express, 14, 11925 Chambouleyron, V., Fauvarque, O., Janin-Potiron, P., et al. 2020, A\&A, 644, A6 Chambouleyron, V., Fauvarque, O., Sauvage, J. F., Neichel, B., \& Fusco, T. 2021, A\&A, 649, A70 
Chanan, G., \& Troy, M. 1999, J. Opt. Soc. Am. A, 38, 6642

Clénet, Y., Buey, T., Gendron, E., et al. 2018, Proc. SPIE, 10703, 10703

Davies, R., Alves, J., Clénet, Y., et al. 2018, Proc. SPIE, 10702, $107021 \mathrm{~S}$

Deo, V., Gendron, E., Rousset, G., et al. 2018, A\&A, 619, A56

Deo, V., Gendron, E., Rousset, G., et al. 2019, A\&A, 629, A107

Deo, V., Gendron, E., Vidal, F., et al. 2021, A\&A, 650, A41

Dierickx, P., \& McPherson, A. 2012, EELT OPTICAL DESIGN REPORT, Tech.

Rep. E-TRE-ESO-313-1000 Issue 2, ESO E-ELT Programme

Engler, B., Le Louarn, M., Vérinaud, C., Weddell, S., \& Clare, R. 2019, in 6st AO4ELT conference-Adaptive Optics for Extremely Large Telescopes

Esposito, S., Riccardi, A., \& Feeney, O. 2000a, Proc. SPIE, 4034, 184

Esposito, S., Feeney, O., \& Riccardi, A. 2000b, Proc. SPIE, 4007, 416

Ferreira, F., Gendron, E., Rousset, G., \& Gratadour, D. 2018, A\&A, 616, A102

Heritier, C. T., Esposito, S., Fusco, T., et al. 2018, MNRAS, 481, 2829

Hippler, S., Feldt, M., Bertram, T., et al. 2019, Exp. Astron., 47, 65

Hutterer, V., Shatokhina, I., Obederer, A., \& Ramlau, R. 2018, J. Astron. Telesc. Instrum. Syst., 4, 049005

Jochum, L., Hubin, N., \& Spyromilio, J. 2008, Technical specifications for the conceptual design, prototyping, preliminary design of the M4 adaptive unit for the E-ELT, Tech. Rep. E-SPE-ESO-106-0037 Issue 3, ESO E-ELT Programme
Korkiakoski, V., Vérinaud, C., Louarn, M. L., \& Conan, R. 2007, Appl. Opt., 46, 6176

Milli, J., Kasper, M., Bourget, P., et al. 2018, Proc. SPIE, 10703, 107032A

N'Diaye, M., Martinache, F., Jovanovic, N., et al. 2018, A\&A, 610, A18

Pinna, E., Esposito, S., Puglisi, A., et al. 2006, Proc. SPIE, 6267, 62672Y

Pinna, E., Quirós-Pacheco, F., Esposito, S., Puglisi, A., \& Stefanini, P. 2008, Proc. SPIE, 7012, 70123D

Ragazzoni, R. 1996, J. Mod. Opt., 43, 289

Sauvage, J. F., Neichel, B., Fusco, T., et al. 2018, Proc. SPIE, 10703

Schwartz, N., Sauvage, J. F., Correia, C., et al. 2018, ArXiv e-prints [arXiv:1809.08839]

Tamai, R., Cirasuolo, M., González, J.C., Koehler, B., \& Tuti, M. 2016, Proc. SPIE, 9906, 99060W

Thatte, N., Tecza, M., Schnetler, H., et al. 2021, Messenger, 182, 7

Vérinaud, C. 2004, Opt. Commun., 233, 27

Vérinaud, C., \& Esposito, S. 2002, in European Southern Observatory Conference and Workshop Proceedings, 58, 153

Vidal, F., Ferreira, F., Deo, V., et al. 2017, in 5th AO4ELT conference-Adaptive Optics for Extremely Large Telescopes

Vievard, S., Bos, S., Cassaing, F., et al. 2019, in 6th AO4ELT conferenceAdaptive Optics for Extremely Large Telescopes

Yaitskova, N., Dohlen, K., \& Dierickx, P. 2003, J. Opt. Soc. Am. A, 20, 1563 


\section{Appendix A: Selection of valid actuators}

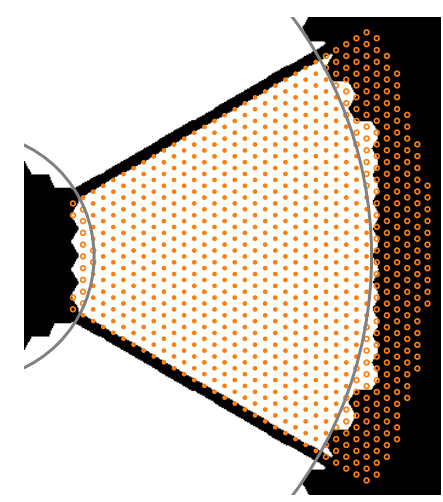

Fig. A.1. Pupil shape and actuator positions within one fragment of the ELT pupil. The active actuators are shown as filled orange dots, located in the active pupil area defined by the two grey circles of $11.4 \mathrm{~m}$ and $37 \mathrm{~m}$ diameters. The empty orange dots are the passive actuators, which were not considered as DM degrees of freedom.

The ELT deformable mirror is made of 5352 actuators, a large number of which are located outside the effective telescope pupil area: four to five actuator rings are located farther away than the outer pupil edges. We selected the actuators that were considered useful degrees of freedom. The selection was made considering the "all-glass" telescope pupil (Dierickx \& McPherson 2012) defined by an inner diameter of 11.4 metres (slightly larger than the $11.2 \mathrm{~m}$ ESO definition), which is the outer diameter of the telescope obstruction; and an outer diameter of 37 metres, which is the inner diameter of the telescope pupil.

The selection is represented in Fig. A.1 for one fragment of the pupil: it gives 642 valid actuators inside the pupil fragment and 250 outside. When replicated to the six fragments of the ELT pupil, we obtain a total of 3852 actuators that we call active actuators and 1500 passive actuators. The first were used to build the modal basis as described in Appendix B, and the second were used in an extension of the modal basis detailed in Appendix C. Some actuators are clearly filtered, but are still inside the effective pupil: We deliberately took some margin in this selection in order to anticipate any actuator divergence. In addition, the performed selection was found to be more robust at all seeing conditions than for instance a selection with a criterion using the true pupil shape (white area of Fig. A.1) or the use of a threshold parameter based on the maximum of the actuator influence function inside the pupil. A better selection depending on the seeing conditions can be performed (a larger number of actuators can be included at small seeing conditions), but this fine-tuning is beyond the scope of this paper.

\section{Appendix B: Definition of the modal basis}

\section{B.1. Construction of the basis}

We defined a modal basis using a light computational method. We call $x_{i}$ the series of positions of the actuators over the pupil. We note $\phi(\boldsymbol{x})$ the vector made of the series of all the $\phi\left(x_{i}\right)$, the phase value at each actuator location. In the case of the ELT adaptive mirror, for which the actuator influence functions are zero-coupled with any other actuator (this not being a property, but a definition), $\phi(x)$ represents both the command vector to the actuators and the wavefront surface at the $x_{i}$ resulting from the application of $\phi(\boldsymbol{x})$.
As a consequence, we propose to compute the principal component analysis (PCA) of the finite-dimension stochastic process of atmospherically perturbed wavefront values $\phi(\boldsymbol{x})$ based on Kolmogorov statistics and deprived from the piston mode (Gendron, priv. comm.). We expect this PCA to naturally yield to some low-order modes extremely close to the well-know Karhunen-Loève infinite expansion of the same continuous process. This avoids the need to go back and forth between continuous and control space, whose computational burden related to sampling problems, heavy arrays, edge effects, etc. is a hassle. The PCA just consists of finding the eigenvector matrix $\boldsymbol{G}$ of the real symmetric covariance matrix $\boldsymbol{C}_{\text {aa }}$ of the piston-subtracted phase process $\phi(\boldsymbol{x})$, such as

$C_{\mathrm{aa}} G=G \lambda$

with

$\boldsymbol{G} \boldsymbol{G}^{T}=\boldsymbol{G}^{T} \boldsymbol{G}=\mathrm{Id}$

To compute $\boldsymbol{C}_{\mathrm{aa}}$, we first computed the spatial covariance of the atmospherically perturbed phase $\varphi$ including the piston term as

$$
\begin{aligned}
\left\langle\varphi\left(x_{i}\right) \varphi\left(x_{j}\right)\right\rangle & =-\frac{1}{2}\left\langle\left(\varphi\left(x_{i}\right)-\varphi\left(x_{j}\right)\right)^{2}\right\rangle+\kappa \\
& =-\frac{1}{2} \mathscr{D}_{\varphi}\left(x_{i j}\right)+\kappa,
\end{aligned}
$$

where $\kappa=\left\langle\varphi^{2}\left(x_{i}\right)+\varphi^{2}\left(x_{j}\right)\right\rangle / 2$ is a constant positive value, independent of $i$ and $j$ because of the spatial invariance properties of $\varphi(x)$. This same property also allows us to introduce the phase structure function $\mathscr{D}_{\varphi}\left(x_{i j}\right)$, applied on the parameter $x_{i j}$ equal to the distance between actuators $i$ and $j$. We note 1 the allone matrix and $n$ the number of actuators. The transition from $\varphi$ (the raw phase including piston) to $\phi$ (centred, piston-subtracted phase) can be made using the centring matrix $\boldsymbol{P}=\mathrm{Id}-\frac{1}{n} \mathbf{1}$ as follows:

$\phi(\boldsymbol{x})=\boldsymbol{P} \varphi(\boldsymbol{x})$.

Noting $\boldsymbol{\Delta} \boldsymbol{x}$ the pairwise actuator distance matrix, using $\mathscr{D}_{\varphi}(\boldsymbol{\Delta x})$ the matrix of the structure function value for each pairwise actuator distance, the expression of the matrix $\boldsymbol{C}_{\mathrm{aa}}$ written in a condensed form becomes

$\boldsymbol{C}_{\mathrm{aa}}=-\frac{1}{2} \boldsymbol{P} \mathscr{D}_{\varphi}(\Delta x) \boldsymbol{P}^{T}$

Note the disappearance of the constant $\kappa$, which vanishes when applying $\boldsymbol{P}$. We are aware that under the assumption of a fully developed Kolmogorov-type turbulence, this constant $\kappa$ has no more mathematical existence than the left-hand member of Eq. B.3. The approach can be justified, however, as the expression B.6 is valid for any arbitrary large finite outer-scale $L_{0}$, therefore remains valid as $L_{0}$ tends towards infinity. We can finally use the expression of the phase structure function $\mathscr{D}_{\varphi}(r)=6.88\left(r / r_{0}\right)^{5 / 3}$. Because we are only interested in the eigenvectors of $\boldsymbol{C}_{\mathbf{a a}}$, the scaling factors do not really matter, and we can finally write

$\boldsymbol{C}_{\mathrm{aa}}=-\boldsymbol{P} \cdot \Delta \boldsymbol{x}^{5 / 3} \cdot \boldsymbol{P}^{T}$

where $\Delta x^{5 / 3}$ is just the matrix filled with the power $5 / 3$ of the actuator pairwise distances. The diagonalisation of $\boldsymbol{C}_{\mathbf{a a}}$ will produce the modes, with a null eigenvalue for the piston mode, of course. 


\section{B.2. Properties of the basis}

This basis offers a series of advantages. Its main attractive feature is its simplicity and very low computational cost. It provides the user with some low-order modes that are nearly identical to those from the projection on the DM of a true, atmospheric Karhunen-Loève basis.

This way of computing modes ignores the exact shape of the influence function and the shape of the pupil. The modes will be orthogonal in the command space only. The pairwise orthogonality of the modes in a phase space (i.e. for the scalar product $\left.\int_{\text {pupil }} m_{i}(r) m_{j}(r) \mathrm{d}^{2} r\right)$ is not guaranteed, but at least the low-order modes will nearly be orthogonal in that sense because the influence functions are spatially local ones. The lack of pairwise phase orthogonality is not an issue because this is not a necessary condition for closing an AO loop with a given basis: The orthogonality in the measurement space would be a greater concern.

Moreover, this basis offers a very interesting property related to the pyramid sensor. The matrix $\mathscr{D}_{\varphi}(\boldsymbol{\Delta x})$ is a convolution matrix that operates over a spatial domain that is sampled by the actuators. The convolution kernel of the operator is the phase structure function: Each column of the matrix is a sampled phase structure function centred on the actuator. The convolution operation is known to become a diagonal one when expressed on a Fourier basis. Therefore the diagonalisation of $\mathscr{D}_{\varphi}(\Delta x)$ will cause some modes to appear whose shape is invariant through a convolution (only the amplitude is affected) and whose frequency content is only made of single-modulus spatial frequencies. The Fourier transform of these modes (or their associated PSF in the small-phase regime) necessarily appears as some thin mono-frequency circles centred on the origin. Because they address a single-frequency modulus, they are particularly suited for forming a basis for optimising the gains with a pyramid sensor.

As a side effect, it is to be noted that this single-modulus frequency property allows us to generalise this technique to any deformable mirror that features identical, circularly symmetric actuator influence functions because the generation by the DM of the wavefront shape associated with a given mode is again a convolution.

The top line of Fig. C.1 shows the obtained modes from the tilt mode up to the last one (pure piston is not considered).

\section{Appendix C: MMSE-based modal extension}

We use a minimum mean-squares error (MMSE) approach to spatially extend some modes, defined over a part of the pupil, to another part. The extension process is achieved according to Kolmogorov statistics. We assumed a wavefront $\phi(x)$ defined through its values at a series of positions $x_{i}$ over the pupil. We wish to estimate its value using a linear relation at some other positions $x_{i}^{\prime}$ in such a way that we minimise the following quantity on average:
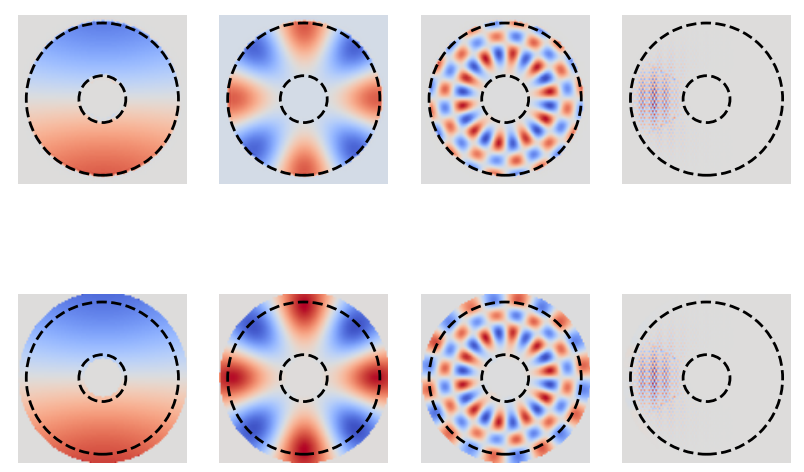

Fig. C.1. DM shape for tilt, fourth-order astigmatism, 100th and last mode defined over the pupil area (top). Modes extended to the actuators located out of the effective pupil (bottom). The dashed circles represent rings that define the active pupil area.

$\left\langle\left|R . \phi(x)-\phi\left(x^{\prime}\right)\right|^{2}\right\rangle$

with $\phi$ following a stochastic process of a Kolmogorov-type perturbed wavefront. The matrix $R$ is the operator we seek. The brackets $\langle\ldots\rangle$ denote the ensemble average or time average. Minimising the mean-squares distance described above allows us to find the wavefront extension that best matches Kolmogorov statistics expectations. The solution of the equation is the socalled MMSE estimator,

$\boldsymbol{R}=\boldsymbol{C}_{\mathrm{pa}} \cdot \boldsymbol{C}_{\mathrm{aa}}{ }^{-1}$.

The matrix $\boldsymbol{C}_{\mathrm{aa}}$ is described in Sect. B. In this section, it is the covariance between the active actuators. Similarly, the matrix $\boldsymbol{C}_{\mathbf{p a}}$ is computed using the same method, between the passive and active actuators.

The matrix $\boldsymbol{R}$ is rectangular with numbers of lines and columns given by the number of passive and the number of active actuators, respectively. We previously computed the modal basis $\boldsymbol{G}$ defined over the active actuators. We can now extend this basis over the other actuators using $\boldsymbol{R}$ in order to span the full DM space. For the sake of clarity, we assumed that the passive actuators are appended as the last elements of the DM command vector. The extended modal basis $\boldsymbol{G}_{\text {extended }}$ is then

$G_{\text {extended }}=\left[\begin{array}{c}G \\ R G\end{array}\right]$.

The bottom line of Fig. C.1 shows the extension of the modes from the active part of the DM to the actuators located outside of the defined ring (shown as the dashed black line). A brief visual analysis shows that the modes are nicely extended, apparently in agreement with Kolmogorov statistics: The tilt mode remains a tilt throughout the DM space, while the estimator has very little effect on the last, high-order mode. 